\title{
Design, Synthesis and Microbiological Evaluation of Novel Compounds as Potential Staphylococcus aureus Phenylalanine tRNA Synthetase Inhibitors
}

\author{
Samar S. Elbaramawi ${ }^{1,2 *}$, Casey Hughes ${ }^{3}$, Jennifer Richards ${ }^{4}$, Arya Gupta ${ }^{5}$, \\ Samy M. Ibrahim ${ }^{2}$, El-Sayed M. Lashine ${ }^{2}$, Mohamed E. El-Sadek², Alex J. \\ O'Neill' ${ }^{5}$, Mandy Wootton ${ }^{4}$, James M. Bullard ${ }^{3}$, Claire Simonss ${ }^{1}$ \\ ${ }^{1}$ School of Pharmacy and Pharmaceutical Sciences, Cardiff University, King Edward \\ VII Avenue, Cardiff CF10 3NB, UK. \\ ${ }^{2}$ Department of Medicinal Chemistry, Faculty of Pharmacy, Zagazig University, \\ Zagazig P.C. 44519, Egypt. \\ ${ }^{3}$ Department of Chemistry, University of Texas - Rio Grande Valley, $1201 \mathrm{~W}$. University \\ Drive, Edinburg, TX 78541, USA. \\ ${ }^{4}$ Specialist Antimicrobial Chemotherapy Unit, University Hospital of Wales, Heath \\ Park, Cardiff CF14 4XW, UK. \\ ${ }^{5}$ School of Molecular \& Cellular Biology, Garstang Building, Faculty of Biological \\ Sciences, University of Leeds, Leeds LS2 9JT, UK.
}

\begin{abstract}
$\mathbf{A}^{\mathrm{s}}$ S THE RESISTANCE of Staphylococcus aureus to antibiotics represents a major threat to global health, anti-infectives with novel mechanisms must be developed. Novel compounds were generated as potential phenylalanine tRNA synthetase (PheRS) inhibitors based on the published homology model of $S$. aureus PheRS to aid the design process using Molecular Operating Environment (MOE) software. PheRS was selected as it is structurally unique enzyme among the aminoacyl-tRNA synthetases (aaRS), it is considerably different from human cytosolic and human mitochondrial aaRS and it is essential and conserved across bacterial species. The designed compounds were synthesized according to different clear schemes. The compounds were confirmed by ${ }^{1} \mathrm{H}$ NMR, ${ }^{13} \mathrm{C}$ NMR, HRMS and/or microanalysis, and they were microbiologically evaluated.
\end{abstract}

Keywords: Staphylococcus aureus, Phenylalanine tRNA synthetase, Drug design, Benzimidazole, Indole, Adenine.

\section{Introduction}

Staphylococcus aureus (S. aureus) commonly colonizes human skin and mucosa without causing any infections. However, if there is an opportunity for the bacteria to enter the body, through broken skin or a medical procedure, they can cause illnesses which range from mild to lifethreatening infections. As they include skin and wound infections, infected eczema, abscesses or joint infections, infections of the heart valves (endocarditis), pneumonia and bacteraemia (blood stream infection). These severe infections acquired either in health-care facilities or in the community [1]. Certain strains of $S$. aureus developed resistance known as methicillin resistant Staphylococcus aureus (MRSA). At present, less than $90 \%$ of $S$. aureus strains are resistant to most penicillin derivatives [2] and ordinary antimicrobial agents like drugs from the family of aminoglycosides, macrolides, chloramphenicols, tetracyclines and fluoroquinolones so known as multidrug resistant Staphylococcus aureus [3].

Increased resistance of MRSA to anti-infective drugs is a threat to global health; so anti-infectives with novel mechanisms must be developed. Our potential target in the drug development for the treatment of MRSA infections is phenylalanine tRNA synthetase which is considered as the most complex and large enzyme of aminoacyl-tRNA synthetases (aaRSs).

Aminoacyl-tRNA synthetases (aaRSs) (also 
known as aminoacyl-tRNA ligases) are essential enzymes for protein biosynthesis, playing a crucial role in the genetic code translation $[4,5]$. AaRSs catalyze the attachment of an amino acid to its cognate tRNA molecule in a two-step reaction. Firstly, cognate amino acids react with ATP forming aminoacyl-adenylate, through a covalent linkage between the 5'-phosphate group of ATP and the carboxyl end of the amino acid. Secondly, the activated forms of the amino acids are subsequently attached to $2^{\prime}-\mathrm{OH}$ or $3^{\prime}-\mathrm{OH}$ of the evolutionarily invariant $3^{\prime}$-adenosine terminus of the cognate tRNA molecule by esterification. The resulting aminoacyl-tRNA acts as a substrate for protein biosynthesis which occurs on ribosomes [4]. The aaRSs are categorized into two classes according to the structural features of the enzymes. Class I enzymes contain a Rossman fold in the catalytic core and two conserved motifs, called HIGH and KMSKS. Class II enzymes have an antiparallel $\beta$-sheet with three conserved motifs in the catalytic centre. To date only one drug, mupirocin, which inhibits a specific type of aaRS (IleRS), has been licensed as a topical antibiotic for the treatment of methicillin-resistant Staphylococcus aureus (MRSA) [6].

Phenylalanine tRNA synthetase (PheRS) is a unique enzyme of the aaRS family, as it is an $(\alpha \beta)_{2}$ heterotetrameric enzyme composed of two small alpha subunits and two larger beta subunits. According to the structure, PheRS is classified as a class II aaRS as its catalytic domain is built around antiparallel $\beta$-sheet but functionally it resembles class I because it aminoacylates the $2^{\prime} \mathrm{OH}$ of the terminal ribose of tRNA where class II aminoacylate the $3^{\prime} \mathrm{OH}[7,8]$. The natural substrate (phenylalanyl-adenylate) was considered as a template for the design of novel potential compounds against $S$. aureus PheRS (Fig.1).

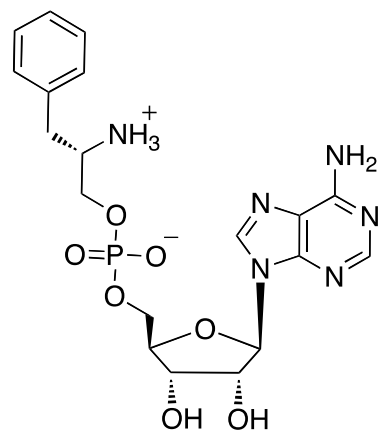

Phenylalanyl-adenylate

Fig. 1. Structures of the natural substrate phenylalanyl-adenylate.

Egypt.J.Chem. Special Issue (2018)

\section{Experimental}

\section{Chemistry}

All employed reagents and solvents were of general purpose or analytical grade and purchased from Fluka, Acros, Alfa-Aeser chemicals and Sigma-Aldrich Chemical Company. Solvents were dried over molecular sieves (4 $\AA$ ). Flash column chromatography was performed with silica gel 60 (Merck 40-60 nm, 230-400 mesh) and Thin layer chromatography (TLC) was performed on precoated silica gel plates (Merck Kiesegel $60 \mathrm{~F}_{254}$ ) with visualization by UV light $(254 \mathrm{~nm})$ and/or vanillin stains. Melting points were determined using Gallenkamp as an electrothermal instrument and they are uncorrected. ${ }^{1} \mathrm{H}$ and ${ }^{13} \mathrm{C}$-NMR spectra were recorded on a Bruker Advance DPX500 spectrometer operating at $500 \mathrm{MHz}$ and $125 \mathrm{MHz}$, respectively. Accurate mass spectroscopic analysis was performed at the EPSRC National Mass Spectrometry Centre (Swansea, UK) and at Medac Ltd., Chobham Business Centre, Surrey, UK. Elemental analysis was performed at Medac Ltd., Chobham Business Centre, Chertsey Road, Surrey, UK.

General method for the synthesis of methyl 3-(1H-benzimidazol-1-yl)propanoate (4a) and methyl 3-(1H-indol-1-yl)propanoate (4b) [9]

To a stirred solution of methylacrylate (3) (3 eq.) and DBU (1 eq.) in acetonitrile $(1.2 \mathrm{~mL} /$ mmol) benzimidazole (1) or indole (2) (2 eq.) was added. The reaction mixture stirred at room temperature for $6 \mathrm{~h}$ in case of benzimidazole and heated at $50{ }^{\circ} \mathrm{C}$ for $6 \mathrm{~h}$ in case of indole. The solvent was evaporated under reduced pressure.

Methyl 3-(1H-benzimidazol-1-yl)propanoate (4a) $\left(\mathrm{C}_{11} \mathrm{H}_{12} \mathrm{~N}_{2} \mathrm{O}_{2}\right.$, M.wt 204.23)

The product was purified by flash column chromatography using dichloromethane: methanol, the product was collected at 98: $2 \%$ v/v. Yield: $8.56 \mathrm{~g}(99 \%)$ as a yellow oil.

TLC: $10 \%$ methanol in dichloromethane, $\mathrm{R}_{\mathrm{f}}=$ 0.61

${ }^{1}$ H NMR (CDCl $)$ ) $\mathbf{~ : ~} 8.03$ (s, 1H, CH-imidazole), $7.83(\mathrm{t}, \mathrm{J}=7.4 \mathrm{~Hz}, 1 \mathrm{H}, \mathrm{Ar}), 7.42(\mathrm{t}, \mathrm{J}=7.6 \mathrm{~Hz}$, 1H, Ar), $7.32(\mathrm{~m}, 2 \mathrm{H}, \mathrm{CH}-\mathrm{Ar}), 4.52(\mathrm{t}, \mathrm{J}=6.5 \mathrm{~Hz}$, $\left.2 \mathrm{H}, \mathrm{CH}_{2}\right), 3.67\left(\mathrm{~s}, 3 \mathrm{H}, \mathrm{CH}_{3}\right), 2.89(\mathrm{t}, \mathrm{J}=6.3 \mathrm{~Hz}$, $2 \mathrm{H}, \mathrm{CH}_{2}$ ).

${ }^{13} \mathbf{C}$ NMR $\left(\mathbf{C D C l}_{3}\right) \boldsymbol{\delta}: 171.04(\mathrm{C}=\mathrm{O}), 143.51(\mathrm{C})$, 143.27 (CH-imidazole), $133.30(\mathrm{C}), 123.27(\mathrm{CH}-$ Ar), 122.41 (CH-Ar), 120.44 (CH-Ar), 109.34 (CH-Ar), $52.13\left(\mathrm{CH}_{3}\right), 40.38\left(\mathrm{CH}_{2}\right), 34.17\left(\mathrm{CH}_{2}\right)$. 
Methyl 3-(1H-indol-1-yl)propanoate (4b) $\left(\mathrm{C}_{12} \mathrm{H}_{13} \mathrm{NO}\right.$, M.wt 203.24)

The product was purified by flash column chromatography using n-hexane: ethylacetate, the product was collected at $90: 10 \% \mathrm{v} / \mathrm{v}$. Yield: 6.94 $\mathrm{g}(99.9 \%)$ as a yellow oil.

TLC: hexane: ethyl acetate, $6: 1, \mathrm{v} / \mathrm{v}, \mathrm{R}_{\mathrm{f}}=0.44$

${ }^{1} \mathbf{H}$ NMR $\left(\mathbf{C D C l}_{3}\right) \boldsymbol{\delta}: 7.85(\mathrm{~d}, \mathrm{~J}=7.9 \mathrm{~Hz}, 1 \mathrm{H}, \mathrm{Ar})$, $7.50(\mathrm{~d}, \mathrm{~J}=8.2 \mathrm{~Hz}, 1 \mathrm{H}, \mathrm{Ar}), 7.44(\mathrm{t}, \mathrm{J}=7.1 \mathrm{~Hz}$, $1 \mathrm{H}, \mathrm{Ar}), 7.37$ (t, J = 7.5 Hz, 1H, Ar), $7.27(\mathrm{~d}, \mathrm{~J}=$ $3.2 \mathrm{~Hz}, 1 \mathrm{H}, \mathrm{Ar}), 6.70(\mathrm{~d}, \mathrm{~J}=3.1 \mathrm{~Hz}, 1 \mathrm{H}, \mathrm{Ar}), 4.52$ $\left(\mathrm{t}, \mathrm{J}=6.9 \mathrm{~Hz}, 2 \mathrm{H}, \mathrm{CH}_{2}\right), 3.79\left(\mathrm{~s}, 3 \mathrm{H}, \mathrm{CH}_{3}\right), 2.90(\mathrm{t}$, $\mathrm{J}=6.9 \mathrm{~Hz}, 2 \mathrm{H}, \mathrm{CH}_{2}$ ).

${ }^{13} \mathbf{C}$ NMR ( $\left.\mathbf{C D C l}_{3}\right) \mathbf{\delta}: 171.78(\mathrm{C}=\mathrm{O}), 135.87(\mathrm{C})$, 128.95 (C), 128.13 (CH-Ar), 121.83 (CH-Ar), 121.25 (CH-Ar), 119.72 (CH-Ar), 109.34 (CHAr), 101.78 (CH-Ar), $51.96\left(\mathrm{CH}_{3}\right), 41.88\left(\mathrm{CH}_{2}\right)$, $34.83\left(\mathrm{CH}_{2}\right)$.

General method for the synthesis of 3-(1H-Benzimidazol-1-yl)propane hydrazide [10,11] (5a) and 3-(1H-Indol-1-yl)propane hydrazide [12] (5b)

To a stirred solution of methyl 3-(1H-benzimidazol-1-yl)propanoate or methyl 3-(1H-indol-1-yl)propanoate (4b) (1 eq.) in methanol $(1 \mathrm{~mL} / \mathrm{mmol})$, hydrazine monohydrate (5 eq.) was added. The reaction mixture was stirred for $3 \mathrm{~h}$ at room temperature, then evaporation of the solvent under vacuum and co-evaporation with diethyl ether to afford solid product. The product was purified by recrystallization form aqueous ethanol.

3-(1H-Benzimidazol-1-yl)propane hydrazide $[10,11](5 a)\left(C_{10} H_{12} \mathrm{~N}_{4} \mathrm{O}\right.$, M.wt 204.23)

Yield: $4.2 \mathrm{~g}(82 \%)$ as yellowish crystals. [Lit. ${ }^{10} 66$ $\%$, Lit. ${ }^{11} 91 \%$ as a white solid].

Melting Point $\left({ }^{\circ} \mathrm{C}\right): 116-118$ [Lit. ${ }^{10} 264-266{ }^{\circ} \mathrm{C}$ ],

TLC: $10 \%$ methanol in dichloromethane, $\mathrm{R}_{\mathrm{f}}=$ 0.57

${ }^{1}$ H NMR (DMSO-d $)$ ) $8: 9.08$ (br s, 1H, NH, $\mathrm{D}_{2} \mathrm{O}-$ exchangeable), 8.13 (s, 1H, CH-imidazole), 7.65 $(\mathrm{d}, \mathrm{J}=8.0 \mathrm{~Hz}, 1 \mathrm{H}, \mathrm{Ar}), 7.60(\mathrm{~d}, \mathrm{~J}=8.0 \mathrm{~Hz}, 1 \mathrm{H}$, Ar), 7.26 (t, J = 7.2 Hz, 1H, Ar), 7.20 (t, J $=7.2$ $\mathrm{Hz}, 1 \mathrm{H}, \mathrm{Ar}), 4.48$ (t, J = 6.6 Hz, 2H, $\left.\mathrm{CH}_{2}\right), 3.93$ (br s, $2 \mathrm{H}, \mathrm{NH}_{2}, \mathrm{D}_{2} \mathrm{O}$-exchangeable), $2.61(\mathrm{t}, \mathrm{J}=$ $6.7 \mathrm{~Hz}, 2 \mathrm{H}, \mathrm{CH}_{2}$ ).

${ }^{13}$ C NMR (DMSO-d $)_{6}$ ): $169.33(\mathrm{C}=\mathrm{O}), 144.50$ (CH-imidazole), 143.83 (C), 134.08 (C), 122.73 (CH-Ar), 121.92 (CH-Ar), 119.85 (CH-Ar),
110.87 (CH-Ar), $44.49\left(\mathrm{CH}_{2}\right), 32.12\left(\mathrm{CH}_{2}\right)$.

3-(1H-Indol-1-yl)propane hydrazide [12] (5b) $\left(C_{11} H_{13} N_{3} O\right.$, M.wt 203.25)

Yield: $3.5 \mathrm{~g}(85 \%)$ as pale-yellow crystals.

Melting Point $\left({ }^{\circ} \mathrm{C}\right)$ : $94-96$

TLC: $10 \%$ methanol in dichloromethane, $\mathrm{R}_{\mathrm{f}}=$ 0.65

${ }^{1} \mathbf{H}$ NMR (DMSO-d $)$ ) 8.0 .04 (s, 1H, NH, $\mathrm{D}_{2} \mathrm{O}-$ exchangeable), $7.53(\mathrm{~d}, \mathrm{~J}=7.9 \mathrm{~Hz}, 1 \mathrm{H}, \mathrm{Ar}), 7.47$ $(\mathrm{d}, \mathrm{J}=8.3 \mathrm{~Hz}, 1 \mathrm{H}, \mathrm{Ar}), 7.29$ (t, J = 2.6 Hz, $1 \mathrm{H}$, Ar), 7.14 (t, J = 7.4 Hz, 1H, Ar), 7.02 (t, J = 7.4 $\mathrm{Hz}, 1 \mathrm{H}, \mathrm{Ar}), 6.41(\mathrm{~d}, \mathrm{~J}=2.3 \mathrm{~Hz}, 1 \mathrm{H}, \mathrm{Ar}), 4.41$ (t, J = 6.8 Hz, 2H, $\mathrm{CH}_{2}$ ), $4.15\left(\mathrm{~s}, 2 \mathrm{H}, \mathrm{NH}_{2}, \mathrm{D}_{2} \mathrm{O}-\right.$ exchangeable), $2.54\left(\mathrm{t}, \mathrm{J}=6.8 \mathrm{~Hz}, 2 \mathrm{H}, \mathrm{CH}_{2}\right)$.

${ }^{13}$ C NMR (DMSO-d $)$ ) $8: 169.63(\mathrm{C}=\mathrm{O}), 135.95$ (C), 129.19 (CH-Ar), 128.59 (C), $121.48(\mathrm{CH}-$ Ar), 121.40 (CH-Ar), 119.42 (CH-Ar), 110.20 (CH-Ar), 101.78 (CH-Ar), $41.84\left(\mathrm{CH}_{2}\right), 34.78$ $\left(\mathrm{CH}_{2}\right)$.

Synthesis of 2-(3-(1H-benzimidazol-1-yl) propanoyl)hydrazine-1-carbothioamide (6a) and 2-(3-(1H-indol-1-yl)propanoyl)hydrazine-1carbothioamide (6b)

A solution of potassium thiocyanate (15.13 $\mathrm{mmol})$ in the least amount of distilled water (2 $\mathrm{mL}), \mathrm{HCl}(1.5 \mathrm{~mL})$ was added dropwise, followed by slow addition of a methanolic solution of 3-(substituted)propane hydrazide (5a, b) (10.05 $\mathrm{mmol}$ ). The reaction mixture was stirred at room temperature overnight. The resulting yellow solid was collected by filtration and washed several times with water. The product was used in the next step without further identification or purification.

Synthesis of 3-(Substituted)-N'-(4-(4-substituted phenyl)thiazol-2-yl)propane hydrazide (8a-c)

Equimolarsolutionsof2-(3-(1H-benzimidazol1-yl)propanoyl)hydrazine-1-carbothioamide (6a) or 2-(3-(1H-indol-1-yl)propanoyl)hydrazine-1carbothioamide (6b) and appropriate 2-bromo-4'substituted acetophenone (7a) or (7b) in absolute ethanol $(20 \mathrm{~mL} / \mathrm{mmol})$ was heated under reflux overnight. The solvent was evaporated under vacuum. The product was purified by flash column chromatography, followed by preparative TLC for final purification.

3-(1H-benzimidazol-1-yl)-N'-(4-(4chlorophenyl)thiazol-2-yl) propane hydrazide (8a) $\left(\mathrm{C}_{19} \mathrm{H}_{16} \mathrm{ClN}_{5} \mathrm{OS}\right.$, M.wt 397.88)

Synthesized using 2-bromo-4'chloroacetophenone (7a) $\quad(0.195 \quad \mathrm{~g}, \quad 0.835$

Egypt.J.Chem. Special Issue (2018) 
mmol). The product was purified by flash column chromatography using dichloromethane: methanol, the product was collected at 93: $7 \%$ $\mathrm{v} / \mathrm{v}$, followed by re-crystallization from ethanol then preparative TLC for final purification using $90 \%$ dichloromethane: $10 \%$ methanol. Yield: $125 \mathrm{mg}(38 \%)$ as a brown solid.

TLC: $10 \%$ methanol in dichloromethane, $\mathrm{R}_{\mathrm{f}}=$ 0.75

${ }^{1}$ H NMR (DMSO-d $)$ 反: 10.28 (s, 1H, NH, $\mathrm{D}_{2} \mathrm{O}$-exchangeable $), 9.50\left(\mathrm{~s}, 1 \mathrm{H}, \mathrm{NH}, \mathrm{D}_{2} \mathrm{O}-\right.$ exchangeable), 8.15 (s, 1H, CH-imidazole), 7.82 (d, J = 8.2 Hz, 2H, Ar), $7.66(\mathrm{t}, \mathrm{J}=9.0 \mathrm{~Hz}, 2 \mathrm{H}$, Ar), 7.44 (d, J = 8.2 Hz, 2H, Ar), 7.24 (m, 3H, 2 $\mathrm{x} \mathrm{Ar}$ and CH-thiazole), $4.56(\mathrm{t}, \mathrm{J}=6.5 \mathrm{~Hz}, 2 \mathrm{H}$, $\left.\mathrm{CH}_{2}\right), 2.80\left(\mathrm{t}, \mathrm{J}=6.5 \mathrm{~Hz}, 2 \mathrm{H}, \mathrm{CH}_{2}\right)$.

${ }^{13}$ C NMR (DMSO-d ) $\boldsymbol{\delta}: 172.66(\mathrm{C}=\mathrm{O}), 170.26$ (C), 149.76 (C), 144.57 (CH-imidazole), 143.93 (C), 134.07 (C), 133.94 (C), 133.41 (C), 132.33 (2 x CH-Ar), 129.08 (2 x CH-Ar), 121.97 (CH-Ar), 121.87 (CH-Ar), 119.90 (CH-Ar), 110.92 (CH-Ar), 104.35 (CH-thiazole), $40.59\left(\mathrm{CH}_{2}\right), 33.93\left(\mathrm{CH}_{2}\right)$.

3 - (1 H-Benzimidazol-1-yl) - N'-(4-(4cyanophenyl)thiazol-2-yl)propane hydrazide (8b) $\left(\mathrm{C}_{20} \mathrm{H}_{16} \mathrm{~N}_{6} \mathrm{OS}\right.$, M.wt 388.45)

Synthesized using 2-bromo-4'-cyanoacetophenone (7b) $(0.43 \mathrm{~g}, 1.89 \mathrm{mmol})$. The product was purified by flash column chromatography using dichloromethane: methanol, the product was collected at $95: 5 \% \mathrm{v} / \mathrm{v}$, followed by preparative TLC for final purification using $90 \%$ dichloromethane : 10 $\%$ methanol. Yield: $153 \mathrm{mg}(21 \%)$ as a yellow solid.

TLC: $10 \%$ methanol in dichloromethane, $\mathrm{R}_{\mathrm{f}}=0.55$

${ }^{1} \mathbf{H}$ NMR (DMSO-d $\left.)_{6}\right) \boldsymbol{\delta}: 10.32$ (s, 1H, NH, $\mathrm{D}_{2} \mathrm{O}$-exchangeable $), 9.60\left(\mathrm{~s}, 1 \mathrm{H}, \mathrm{NH}, \mathrm{D}_{2} \mathrm{O}-\right.$ exchangeable), 8.15 (s, 1H, CH-imidazole), 7.97 (d, J = 8.4 Hz, 2H, Ar), 7.83 (d, J = 8.4 Hz, 2H, Ar), 7.66 (dd, J = 8.0, $13.3 \mathrm{~Hz}, 2 \mathrm{H}, \mathrm{Ar}), 7.51$ (s, 1H, CH-thiazole), 7.25 (m, 2H, Ar), 4.55 (t, J = $\left.6.3 \mathrm{~Hz}, 2 \mathrm{H}, \mathrm{CH}_{2}\right), 2.81\left(\mathrm{t}, \mathrm{J}=6.2 \mathrm{~Hz}, 2 \mathrm{H}, \mathrm{CH}_{2}\right)$.

${ }^{13}$ C NMR (DMSO-d ) $\boldsymbol{\delta}: 172.83(\mathrm{C}=\mathrm{O}), 170.29$ (C), 149.27 (C), 144.57 (CH-imidazole), 143.94 (C), 139.15 (C), 134.08 (C), 133.10 (2 x CH-Ar), 126.64 (2 x CH-Ar), 121.98 (CH-Ar), 121.87 (CH-Ar), 119.91 (CH-Ar), $119.46(\mathrm{CN}), 110.92$ (CH-Ar), 110.02 (C), 107.40 (CH-thiazole), 40.59 $\left(\mathrm{CH}_{2}\right), 33.92\left(\mathrm{CH}_{2}\right)$.

$N^{\prime}$-(4-(4-Cyanophenyl)thiazol-2-yl)-3-(1Hindol-1-yl)propanehydarzide (8c) $\left(\mathrm{C}_{21} \mathrm{H}_{17} \mathrm{~N}_{5} \mathrm{OS}\right.$, M.wt 387.46)

Egypt.J.Chem. Special Issue (2018)
Synthesized using 2-bromo-4'-cyanoacetophenone (7b) (0.26 g, $1.14 \mathrm{mmol})$. The product was purified by flash column chromatography using petroleum ether: ethylacetate, the product was collected at 40: $60 \%$ v/v. Yield: $172 \mathrm{mg}$ (39 $\%$ ) as a yellow solid.

TLC: petroleum ether : ethyl acetate $1: 4, \mathrm{v} / \mathrm{v}, \mathrm{R}_{\mathrm{f}}$ $=0.66$

${ }^{1}$ H NMR (DMSO-d) d: $_{10.29}$ (s, 1H, NH, $\mathrm{D}_{2} \mathrm{O}$-exchangeable), $9.59\left(\mathrm{~s}, 1 \mathrm{H}, \mathrm{NH}, \mathrm{D}_{2} \mathrm{O}-\right.$ exchangeable), 8.00 (d, J = 8.2 Hz, 2H, Ar), 7.85 (d, J = 8.2 Hz, 2H, Ar), 7.57 (d, J = 7.5 Hz, 2H, Ar), 7.52 (s, 1H, CH-thiazole), $7.50(\mathrm{~d}, \mathrm{~J}=7.5 \mathrm{~Hz}$, $2 \mathrm{H}, \mathrm{Ar}), 7.33$ (s, 1H, Ar), 7.15 (t, J = $7.2 \mathrm{~Hz}, 1 \mathrm{H}$, Ar), 7.03 (t, J = 7.2 Hz, 1H, Ar), 6.43 (s, 1H, Ar), $4.47\left(\mathrm{t}, \mathrm{J}=6.6 \mathrm{~Hz}, 2 \mathrm{H}, \mathrm{CH}_{2}\right), 2.72(\mathrm{t}, \mathrm{J}=6.6 \mathrm{~Hz}$, $\left.2 \mathrm{H}, \mathrm{CH}_{2}\right)$.

${ }^{13}$ C NMR (DMSO-d $)$ ) $\mathbf{\delta}: 172.89(\mathrm{C}=\mathrm{O}), 170.57$ (C), 149.25 (C), 139.15 (C), 135.95 (C), 133.11 (2 x CH-Ar), 129.04 (CH-Ar), 128.67 (C), 126.63 ( 2 x CH-Ar), 121.42 (CH-Ar), 120.90 (CH-Ar), 119.51 (CH-Ar), 119.46 (CN), 110.22 (CH-Ar), 110.06 (C), 107.41 (CH-thiazole), 101.25 (CHAr), $41.31\left(\mathrm{CH}_{2}\right), 34.53\left(\mathrm{CH}_{2}\right)$.

Synthesis of 5-(2-(1H-benzimidazol-1-yl)ethyl)1,3,4-thiadiazol-2-amine (9) $\left(\mathrm{C}_{11} \mathrm{H}_{11} \mathrm{~N}_{5} \mathrm{~S}, \quad \mathrm{M} . w \mathrm{t}\right.$ 245.07)

Potassium thiocyanate $(1.07 \mathrm{~g}, 11.02 \mathrm{mmol})$ was dissolved in the least amount of water (2 $\mathrm{mL})$ then hydrochloric acid $(1 \mathrm{~mL})$ was added dropwise. The aforementioned mixture was added to a methanolic solution of 3-(1H-benzimidazol1-yl)propane hydrazide (5a) (1.5 g, $7.34 \mathrm{mmol})$. The reaction mixture was stirred overnight at room temperature, followed by solvent evaporation under vacuum. The resulting solid was added portionwise to $\mathrm{H}_{2} \mathrm{SO}_{4}(5 \mathrm{~mL})$ with continuous stirring. The reaction mixture was stirred for $2 \mathrm{~h}$, then slowly poured into crushed ice with stirring and neutralized with ammonia solution. The resulting pale brown solid was collected by filtration under vacuum. The product was pure enough to proceed to further reaction. Yield: $1.5 \mathrm{~g}$ $(88 \%)$ as a pale-brown solid.

\section{Melting Point ( $\left.{ }^{\circ} \mathrm{C}\right): 182-184$}

TLC: $10 \%$ methanol in dichloromethane, $\mathrm{R}_{\mathrm{f}}=$ 0.37

${ }^{1}$ H NMR (DMSO-d $)$ ): $8.16(\mathrm{~s}, 1 \mathrm{H}, \mathrm{CH}-$ imidazole), 7.63 (t, J = 7.5 Hz, 2H, Ar), 7.26 (t, $\mathrm{J}=7.3 \mathrm{~Hz}, 1 \mathrm{H}, \mathrm{Ar}), 7.21$ (t, J = 7.5 Hz, 1H, Ar), 
$7.03\left(\mathrm{~s}, 2 \mathrm{H}, \mathrm{NH}_{2}, \mathrm{D}_{2} \mathrm{O}\right.$-exchangeable $), 4.60(\mathrm{t}, \mathrm{J}=$ $\left.6.7 \mathrm{~Hz}, 2 \mathrm{H}, \mathrm{CH}_{2}\right), 3.40\left(\mathrm{t}, \mathrm{J}=6.7 \mathrm{~Hz}, 2 \mathrm{H}, \mathrm{CH}_{2}\right)$.

${ }^{13}$ C NMR (DMSO-d $)$ ) $: 169.15(\mathrm{C}), 154.69(\mathrm{C})$, 144.61 (CH-imidazole), 143.89 (C), 134.12 (C), 122.81 (CH-Ar), 121.99 (CH-Ar), 119.92 (CHAr), 110.90 (CH-Ar), $43.68\left(\mathrm{CH}_{2}\right), 30.32\left(\mathrm{CH}_{2}\right)$

[ESI-HRMS] Calculated mass: 246.0726 $[\mathrm{M}+\mathrm{H}]^{+}$, Measured mass: $246.0720[\mathrm{M}+\mathrm{H}]^{+}$.

General method for the synthesis of $\mathrm{N}-(5-(2-(1 H-$ benzimidazol-1-yl)ethyl)-1,3,4-thiadiazol-2-yl)substituted benzamide (11a, b)

To a solution of 5-(2-(1H-benzimidazol1-yl)ethyl)-1,3,4-thiadiazol-2-amine (9) (1 eq.) in dry dichloromethane $(10 \mathrm{~mL} / \mathrm{mmol})$, triethylamine (10 eq.) was added. The reaction mixture was cooled to $0{ }^{\circ} \mathrm{C}$, followed by addition of 3,5-dimethoxybenzoyl chloride (10a) or 4-fluorobenzoyl chloride (10b) (1.1 eq.) in dry dichloromethane $(10 \mathrm{~mL})$ dropwise over $30 \mathrm{~min}$. Then, the reaction mixture was stirred at room temperature overnight. Solvent was evaporated under pressure and the resulting solid was extracted with dichloromethane $(50 \mathrm{~mL} / \mathrm{mmol})$ and saturated aqueous sodium bicarbonate (3 x $25 \mathrm{~mL} / \mathrm{mmol}$ ). The organic layer was dried over anhydrous magnesium sulfate, filtered and evaporated under reduced pressure. The product was purified by flash column chromatography using gradient elution of dichloromethane: methanol, the product was collected at 96: $4 \%$ $\mathrm{v} / \mathrm{v}$.

$N-(5-(2-(1 H-b e n z i m i d a z o l-1-y l)$ ethyl)-1,3,4thiadiazol-2-yl)-3,5-dimethoxybenzamide (11a) $\left(\mathrm{C}_{20} \mathrm{H}_{19} \mathrm{~N}_{5} \mathrm{O}\right.$ S, M.wt 409.46)

Yield: $0.15 \mathrm{~g}(56 \%)$ as a white solid.

Melting Point ( $\left.{ }^{\circ} \mathbf{C}\right):$ 200-202

TLC: $10 \%$ methanol in dichloromethane, $\mathrm{R}_{\mathrm{f}}=$ 0.6

${ }^{\mathbf{1}} \mathbf{H}$ NMR (DMSO-d $)$ ) 12.95 (s, 1H, NH, D 2 exchangeable), 8.18 (s, 1H, CH-imidazole), 7.66 (t, J = 8.3 Hz, 2H, Ar), 7.25 (m, 4H, Ar), 6.76 (s, $1 \mathrm{H}, \mathrm{Ar}), 4.72\left(\mathrm{t}, \mathrm{J}=6.8 \mathrm{~Hz}, 2 \mathrm{H}, \mathrm{CH}_{2}\right), 3.82(\mathrm{~s}, 6 \mathrm{H}$, $\left.2 \times \mathrm{CH}_{3}\right), 3.62\left(\mathrm{t}, \mathrm{J}=6.8 \mathrm{~Hz}, 2 \mathrm{H}, \mathrm{CH}_{2}\right)$.

13C NMR (DMSO-d6) $\boldsymbol{\delta}: 165.10(\mathrm{C}=\mathrm{O}), 161.11$ (C), 160.94 ( $2 \times$ C), 160.17 (C), 144.57 (C), 143.90 (CH-imidazole), 134.33 (C), 133.83 (C), 129.11 (CH-Ar), 122.84 (CH-Ar), 122.04 (CHAr), 119.95 (CH-Ar), 110.91 (CH-Ar), 106.47 (CH-Ar), 105.79 (CH-Ar), $55.06\left(\mathrm{CH}_{3}\right), 43.76$ $\left(\mathrm{CH}_{2}\right), 29.94\left(\mathrm{CH}_{2}\right)$.
[ESI-HRMS] Calculated mass: 410.1281 $[\mathrm{M}+\mathrm{H}]^{+}$, Measured mass: $410.1278[\mathrm{M}+\mathrm{H}]^{+}$.

$N-(5-(2-(1 H-b e n z i m i d a z o l-1-y l) e t h y l)-$ 1,3,4-thiadiazol-2-yl)-4-fluorobenzamide

(11b) ( $\mathrm{C}_{18} \mathrm{H}_{14} \mathrm{FN}_{5} \mathrm{OS}$, M.wt 367.40)

Yield: $0.4 \mathrm{~g}(45 \%)$ as a yellow solid.

\section{Melting Point ( $\left.{ }^{\circ} \mathrm{C}\right):$ 210-212}

TLC: $10 \%$ methanol in dichloromethane, $\mathrm{R}_{\mathrm{f}}=$ 0.8

${ }^{1}$ H NMR (DMSO-d $) \boldsymbol{\delta}: 13.03$ (s, 1H, NH, D Oexchangeable), $8.17(\mathrm{t}, \mathrm{J}=7.5 \mathrm{~Hz}, 3 \mathrm{H}, 2 \mathrm{Ar}$ and CH-imidazole), 8.01 (d, J = 8.8 Hz, 1H, Ar), 7.66 $(\mathrm{d}, \mathrm{J}=8.0 \mathrm{~Hz}, 1 \mathrm{H}, \mathrm{Ar}), 7.27$ (m, 4H, Ar), 4.72 (t, J $=6.8 \mathrm{~Hz}, 2 \mathrm{H}, \mathrm{CH} 2), 3.62\left(\mathrm{t}, \mathrm{J}=6.8 \mathrm{~Hz}, 2 \mathrm{H}, \mathrm{CH}_{2}\right)$.

${ }^{13}$ C NMR (DMSO-d $)$ ) $8: 166.84(\mathrm{C}=\mathrm{O}), 166.38$ (C), 164.39 (C), 161.07 (C), $143.90 \quad(\mathrm{CH}-$ imidazole), $143.88(\mathrm{C}), 134.07(\mathrm{C}), 132.61(\mathrm{CH}-$ Ar), 132.54 (CH-Ar), 128.67 (C), 122.84 (CHAr), 122.04 (CH-Ar), 119.94 (CH-Ar), 116.18 (CH-Ar), 116.00 (CH-Ar), 110.91 (CH-Ar), 43.73 $\left(\mathrm{CH}_{2}\right), 29.96\left(\mathrm{CH}_{2}\right)$.

[ESI-HRMS] Calculated mass: 368.0976 $[\mathrm{M}+\mathrm{H}]^{+}$, Measured mass: $368.0977[\mathrm{M}+\mathrm{H}]^{+}$.

General procedures for the synthesis of 5-(3-(1H-Benzimidazol-1-yl)propyl)-4-phenyl2,4-dihydro-3H-1,2,4-triazole-3-thione (14) and 5-(3-(6-Amino-9H-purin-9-yl)propyl)-4-phenyl2,4-dihydro-3H-1,2,4-triazole-3-thione (15)

To a suspension of 2-(4-(1H-benzimidazol1 -yl)butanoy 1$)-N$-phenylhydrazine - 1 carbothioamide (12) or 2-(4-(6-amino- $9 H$ purin-9-yl)butanoyl)- $N$-phenylhydrazine-1carbothioamide (13) (1 eq.) in ethanol $(15 \mathrm{~mL} /$ mmol) was added $2 \mathrm{~N}$ aqueous $\mathrm{NaOH}(5 \mathrm{~mL} /$ mmol) dropwise with continuous stirring. The reaction mixture was stirred at room temperature for $5 \mathrm{~h}$. Evaporation of ethanol under reduced pressure. The solution was neutralized by the addition of $\mathrm{HCl}$ dropwise until the formation of a white precipitate. The precipitate was collected by filtration under vacuum.

5-(3-(1H-Benzimidazol-1-yl)propyl)-4phenyl-2,4-dihydro-3H-1,2,4-triazole-3-thione (14) $\left(C_{18} H_{17} N_{5} S\right.$, M.wt 335.43)

The product was purified by flash column chromatography using dichloromethane: methanol, the product was collected at 96: $4 \%$ v/v. Yield: $0.204 \mathrm{~g}(72 \%)$ as a white solid.

Melting Point $\left({ }^{\circ} \mathbf{C}\right): 98-100$ 
TLC: $10 \%$ methanol in dichloromethane, $\mathrm{R}_{\mathrm{f}}=$ 0.52

${ }^{1}$ H NMR (DMSO-d $)$ $\boldsymbol{\delta}: 13.61$ (br s, $1 \mathrm{H}, \mathrm{NH}$, $\mathrm{D}_{2} \mathrm{O}$-exchangeable), 8.04 (s, 1H, CH-imidazole), 7.92 (d, J = 6.9 Hz, 1H, Ar), 7.48 (d, J = 6.5 Hz, $3 \mathrm{H}, \mathrm{Ar}), 7.31(3 \mathrm{H}, \mathrm{J}=6.7 \mathrm{~Hz}, \mathrm{Ar}), 7.20(\mathrm{t}, \mathrm{J}=3.0$ $\mathrm{Hz}, \mathrm{Ar}), 4.30$ (t, J = 6.7 Hz, 2H, $\left.\mathrm{CH}_{2}\right), 2.48(\mathrm{t}, \mathrm{J}$ $=6.9 \mathrm{~Hz}, 2 \mathrm{H}, \mathrm{CH}_{2}$ ), 2.21 (quin., $\mathrm{J}=6.9 \mathrm{~Hz}, 2 \mathrm{H}$, $\mathrm{CH}_{2}$ ).

${ }^{13}$ C NMR (DMSO-d $) \boldsymbol{\delta}: 168.90$ (C), 151.07 (C), 142.98 (CH-imidazole), 144.00 (C), 133.47 (C), 133.06 (C), 130.22 (2 x CH-Ar), 130.01 (2 x CHAr), 127.65 (CH-Ar), 123.26 (CH-Ar), 122.50 (CH-Ar), 120.59 (CH-Ar), 109.44 (CH-Ar), 43.43 $\left(\mathrm{CH}_{2}\right), 25.58\left(\mathrm{CH}_{2}\right), 22.79\left(\mathrm{CH}_{2}\right)$.

[ESI-HRMS] Calculated mass: 336.1277 $[\mathrm{M}+\mathrm{H}]^{+}$, Measured mass: $336.1284[\mathrm{M}+\mathrm{H}]^{+}$.

5-(3-(6-Amino-9H-purin-9-yl)propyl)-4phenyl-2,4-dihydro-3H-1,2,4-triazole-3-thione (15) $\left(C_{16} H_{16} N_{8} S\right.$, M.wt 352.42)

The product was purified by re-crystallization from aqueous ethanol. Yield: $2.3 \mathrm{~g}(97 \%)$ as white crystals.

\section{Melting Point $\left({ }^{\circ} \mathbf{C}\right):$ 280-282}

TLC: $10 \%$ methanol in dichloromethane, $\mathrm{R}_{\mathrm{f}}=$ 0.4

${ }^{1}$ H NMR (DMSO-d $)$ ) $\boldsymbol{\delta}: 13.73$ (s, 1H, NH, D 2 exchangeable), 8.09 (s, 1H, CH-imidazole), 8.03 (s, 1H, CH-pyrimidine), 7.49 (d, J = 6.5 Hz, 3H, $\mathrm{Ar}), 7.35$ (d, J = 7.6 Hz, 2H, Ar), 7.17 (s, 2H, NH, $\mathrm{D}_{2} \mathrm{O}$-exchangeable), $4.15\left(\mathrm{t}, \mathrm{J}=6.6 \mathrm{~Hz}, 2 \mathrm{H}, \mathrm{CH}_{2}\right)$, $2.41\left(\mathrm{t}, \mathrm{J}=7.3 \mathrm{~Hz}, 2 \mathrm{H}, \mathrm{CH}_{2}\right.$ ), 2.06 (quin., $\mathrm{J}=6.8$ $\mathrm{Hz}, 2 \mathrm{H}, \mathrm{CH}_{2}$ ).

${ }^{13}$ C NMR (DMSO-d) d: $^{172.91}$ (C), 161.15 (C), 157.56 (CH-pyrimidine), 156.48 (C), 154.68 (C), 141.33 (CH-imidazole), 138.76 (C), 134.60 (2 x CH-Ar), 134.51 (2 x CH-Ar), 133.35 (CHAr), $123.94(\mathrm{C}), 47.13\left(\mathrm{CH}_{2}\right), 30.68\left(\mathrm{CH}_{2}\right), 27.77$ $\left(\mathrm{CH}_{2}\right)$.

[ESI-HRMS] Calculated mass: 353.1291 $[\mathrm{M}+\mathrm{H}]^{+}$, Measured mass: $353.1291[\mathrm{M}+\mathrm{H}]^{+}$.

General procedure for the synthesis of 1-(3-(4-phenyl-5-(substituted thio)-4H-1,2,4triazol-3-yl)propyl)1H-benzimidazole (16a,b).

To a mixture of 5-(3-(1H-benzimidazol-1-yl) propyl)-4-phenyl-2,4-dihydro-3H-1,2,4-triazole3-thione (14) (1 eq.) and anhydrous potassium carbonate (1.5 eq.) in dry DMF $(10 \mathrm{~mL} / 0.5 \mathrm{mmol})$ iodoethane or 1-iodopropane (1 eq.) in dry DMF $(5 \mathrm{~mL} / 0.5 \mathrm{mmol})$ was added. The reaction mixture was stirred overnight at room temperature. The reaction mixture was concentrated under vacuum and the residue was dissolved in ethyl acetate $(100 \mathrm{~mL} / 0.5 \mathrm{mmol})$ and washed with water $(3 \mathrm{x}$ $50 \mathrm{~mL} / 0.5 \mathrm{mmol})$. The organic layer was dried over anhydrous $\mathrm{MgSO}_{4}$ and concentrated under vacuum.

1-(3-(5-(Ethylthio)-4-phenyl-4H-1,2,4triazol-3-yl)propyl)-1H-benzimidazole

(16a) $\left(\mathrm{C}_{20} \mathrm{H}_{21} \mathrm{~N}_{5} \mathrm{~S}\right.$, M.wt 363.48)

The product was purified by flash column chromatography using gradient elution of dichloromethane: methanol, the product was collected at 94: $6 \% \mathrm{v} / \mathrm{v}$. Yield: $0.2 \mathrm{~g}(84 \%)$ as a yellowish solid.

\section{Melting Point ( $\left.{ }^{\circ} \mathrm{C}\right): 118-120$}

TLC: $10 \%$ methanol in dichloromethane, $\mathrm{R}_{\mathrm{f}}=$ 0.6

${ }^{1} \mathbf{H}$ NMR (CDCl $) \boldsymbol{~} \boldsymbol{\delta}: 7.85$ (s, 1H, CH-imidazole), $7.78(\mathrm{~d}, \mathrm{~J}=6.9 \mathrm{~Hz}, 1 \mathrm{H}, \mathrm{Ar}), 7.48: 7.42(\mathrm{~m}, 3 \mathrm{H}$, Ar), 7.29 (m, 3H, Ar), $7.04(\mathrm{~d}, \mathrm{~J}=7.1 \mathrm{~Hz}, 1 \mathrm{H}$, Ar), $4.41\left(\mathrm{t}, \mathrm{J}=6.7 \mathrm{~Hz}, 2 \mathrm{H}, \mathrm{CH}_{2}\right), 3.23$ (q, J = 7.3 $\left.\mathrm{Hz}, 2 \mathrm{H}, \mathrm{CH}_{2}\right), 2.48$ (t, J = 6.9 Hz, 2H, $\left.\mathrm{CH}_{2}\right), 2.29$ (quin., $\mathrm{J}=6.6 \mathrm{~Hz}, 2 \mathrm{H}, \mathrm{CH}_{2}$ ), $1.41(\mathrm{t}, \mathrm{J}=7.3 \mathrm{~Hz}$, $\left.3 \mathrm{H}, \mathrm{CH}_{3}\right)$.

${ }^{13} \mathbf{C}$ NMR (CDCl $)$ d: 154.13 (C), 151.90 (C), 143.86 (C), 143.04 (CH-imidazole), 133.73 (C), 132.90 (C), 130.09 (2 x CH-Ar), 130.02 (2 x CHAr), 126.82 (CH-Ar), 122.93 (CH-Ar), 122.10 (CH-Ar), 120.39 (CH-Ar), 109.68 (CH-Ar), 43.40 $\left(\mathrm{CH}_{2}\right), 26.88\left(\mathrm{CH}_{2}\right), 21.79\left(\mathrm{CH}_{2}\right), 14.77\left(\mathrm{CH}_{3}\right)$.

Microanalysis: Theoretical: \%C: $66.09, \% \mathrm{H}$ : $5.82, \% \mathrm{~N}: 19.26$, Found: \%C: $65.64, \% \mathrm{H}: 5.74$, $\% \mathrm{~N}: 19.09$.

1-(3-(4-Phenyl-5-(propylthio)-4H-1,2,4triazol-3-yl)propyl)1H-benzimidazole

(16b) $\left(C_{21} H_{23} N_{5} S\right.$, M.wt: 377.51)

The product was purified by flash column chromatography using gradient elution of dichloromethane: methanol, the product was collected at 97: $3 \% \mathrm{v} / \mathrm{v}$. Yield: $0.27 \mathrm{~g}(80 \%)$ as a yellow oil.

TLC: $10 \%$ methanol in dichloromethane, $\mathrm{R}_{\mathrm{f}}=$ 0.6

${ }^{1} \mathbf{H}$ NMR (CDCl $)$ ) $\mathbf{~ : ~} 7.86$ (s, 1 H, CH-imidazole), $7.76(\mathrm{~d}, 1 \mathrm{H}, \mathrm{J}=6.7, \mathrm{Ar}), 7.41: 7.47$ (m, 3H, Ar), 7.29 (d, J = 7.6, 1H, Ar), 7.22: 7.26 (m, 3H, Ar), 
$7.02(\mathrm{~d}, \mathrm{~J}=7.0,2 \mathrm{H}, \mathrm{Ar}), 4.38(\mathrm{t}, \mathrm{J}=6.7,3 \mathrm{H}$, $\left.\mathrm{CH}_{2}\right), 3.17\left(\mathrm{t}, \mathrm{J}=7.3,3 \mathrm{H}, \mathrm{CH}_{2}\right), 2.47(\mathrm{t}, \mathrm{J}=6.7$, $3 \mathrm{H}, \mathrm{CH}_{2}$ ), 2.27 (quin., $\mathrm{J}=6.6 \mathrm{~Hz}, 2 \mathrm{H}, \mathrm{CH}_{2}$ ), 1.75 $\left(\mathrm{m}, 2 \mathrm{H}, \mathrm{CH}_{2}\right), 0.98\left(\mathrm{t}, \mathrm{J}=7.2,3 \mathrm{H}, \mathrm{CH}_{3}\right)$

${ }^{13} \mathbf{C}$ NMR $\left(\mathbf{C D C l}_{3}\right) \boldsymbol{\delta}: 154.12(\mathrm{C}), 152.12(\mathrm{C})$, 143.69 (C), 143.02 (CH-imidazole), 133.69 (C), 132.88 (C), 130.08 (2 x CH-Ar), 130.01 ( 2 x CHAr), 126.82 (CH-Ar), 122.96 (CH-Ar), 122.14 (CH-Ar), 120.29 (CH-Ar), 109.72 (CH-Ar), 43.43 $\left(-\mathrm{CH}_{2}\right), 34.42\left(\mathrm{CH}_{2}\right), 26.30\left(\mathrm{CH}_{2}\right), 22.75\left(\mathrm{CH}_{2}\right)$, $21.79\left(\mathrm{CH}_{2}\right), 13.24(\mathrm{CH} 3)$.

[ESI-HRMS] Calculated mass: 378.1747 $[\mathrm{M}+\mathrm{H}]^{+}$, Measured mass: $378.1749[\mathrm{M}+\mathrm{H}]^{+}$.

General procedure for the synthesis of 9-(3-(4-phenyl-5-(substituted thio)-4H-1,2,4triazol-3-yl)propyl)-9H-purin-6-amine $(17 a, b)$

To a mixture of 5-(3-(6-amino-9H-purin-9-yl) propyl)-4-phenyl-2,4-dihydro-3H-1,2,4-triazole3-thione (15) (1 eq.) and anhydrous potassium carbonate (1.5 eq.) in dry DMF $(10 \mathrm{~mL} / 0.5 \mathrm{mmol})$ iodoethane or 1 -iodopropane (1 eq.) in dry DMF $(5 \mathrm{~mL} / 0.5 \mathrm{mmol})$ was added. The reaction mixture was stirred overnight at room temperature. The reaction mixture was concentrated under vacuum and the residue was dissolved in ethyl acetate $(100 \mathrm{~mL} / 0.5 \mathrm{mmol})$ and washed with water $(3 \mathrm{x}$ $50 \mathrm{~mL} / 0.5 \mathrm{mmol})$. The organic layer was dried over anhydrous $\mathrm{MgSO}_{4}$ and concentrated under vacuum.

9-(3-(5-(Ethylthio)-4-phenyl-4H-1,2,4triazol-3-yl)propyl)-9H-purin-6-amine (17a) $\left(C_{18} H_{20} N_{8} S\right.$, M.wt 380.47)

The product was purified by flash column chromatography using gradient elution of dichloromethane: methanol, the product was collected at 92: $8 \% \mathrm{v} / \mathrm{v}$. Yield: $0.6 \mathrm{~g}(86 \%)$ as yellow crystals.

\section{Melting Point $\left({ }^{\circ} \mathrm{C}\right): 180-182^{\circ} \mathrm{C}$}

TLC: $10 \%$ methanol in dichloromethane, $\mathrm{R}_{\mathrm{f}}=0.3$

${ }^{\mathbf{1}} \mathbf{H}$ NMR (DMSO-d) $) \quad \boldsymbol{\delta}: 8.09$ (s, 1H, CHpyrimidine), 8.04 (s, 1H, CH-imidazole), 7.51 (m, $3 \mathrm{H}, \mathrm{Ar}), 7.35$ (m, 2H, Ar), $7.18\left(\mathrm{~s}, 2 \mathrm{H}, \mathrm{NH}_{2}\right), 4.17$ $\left(\mathrm{t}, \mathrm{J}=6.8,2 \mathrm{H}, \mathrm{CH}_{2}\right), 3.04\left(\mathrm{q}, \mathrm{J}=7.4 \mathrm{~Hz}, 2 \mathrm{H}, \mathrm{CH}_{2}\right.$ ), 2.52 (masked by DMSO peak, $\mathrm{CH}_{2}$ ), 2.12 (quin., $\left.\mathrm{J}=6.7 \mathrm{~Hz}, 2 \mathrm{H}, \mathrm{CH}_{2}\right), 1.26\left(\mathrm{t}, \mathrm{J}=7.4,3 \mathrm{H}, \mathrm{CH}_{3}\right)$.

${ }^{13}$ C NMR (DMSO-d $)$ ) $\boldsymbol{\delta}: 156.40$ (C), 154.97 (C), 152.78 (CH-Ar), 150.25 (C), 149.93 (C), 141.20 (CH-Ar), 133.46 (C), 130.28 (2 x CH-Ar), 130.22 (2 x CH-Ar), 127.65 (CH-Ar), 119.23 (C), 42.62
$\left(\mathrm{CH}_{2}\right), 27.03\left(\mathrm{CH}_{2}\right), 26.85\left(\mathrm{CH}_{2}\right), 22.36\left(\mathrm{CH}_{2}\right)$, $15.28\left(\mathrm{CH}_{3}\right)$.

Microanalysis: Theoretical: \%C: $56.82, \% \mathrm{H}$ : $5.30, \% \mathrm{~N}: 29.44$, Found: \%C: $56.33, \% \mathrm{H}: 5.22$, $\% \mathrm{~N}: 28.99$.

9-(3-(4-Phenyl-5-(propylthio)-4H-1,2,4triazol-3-yl)propyl)-9H-purin-6-amine (17b) $\left(\mathrm{C}_{19} \mathrm{H}_{22} \mathrm{~N}_{8} \mathrm{~S}\right.$, M.wt 394.50)

The product was purified by flash column chromatography using gradient elution of dichloromethane: methanol, the product was collected at 94: $6 \% \mathrm{v} / \mathrm{v}$. Yield: $0.64 \mathrm{~g}(88 \%)$ as white crystals.

\section{Melting Point $\left({ }^{\circ} \mathrm{C}\right): 174-178$}

TLC: $10 \%$ methanol in dichloromethane, $\mathrm{R}_{\mathrm{f}}=$ 0.58

${ }^{1} \mathbf{H}$ NMR (DMSO-d $) \quad \boldsymbol{\delta}: 8.10(\mathrm{~s}, 1 \mathrm{H}, \mathrm{CH}-$ pyrimidine), 8.05 (s, $1 \mathrm{H}, \mathrm{CH}$-imidazole), 7.51 (m, $3 \mathrm{H}, \mathrm{Ar}), 7.35$ (m, 2H, Ar), 7.20 (s, 2H, $\left.\mathrm{NH}_{2}\right), 4.17$ $\left(\mathrm{t}, \mathrm{J}=6.7,2 \mathrm{H}, \mathrm{CH}_{2}\right), 3.02\left(\mathrm{t}, \mathrm{J}=6.9,2 \mathrm{H}, \mathrm{CH}_{2}\right)$, $2.52\left(\mathrm{t}, \mathrm{J}=7.6,2 \mathrm{H}, \mathrm{CH}_{2}\right.$ ), 2.12 (quin., $\mathrm{J}=6.6 \mathrm{~Hz}$, $\left.2 \mathrm{H}, \mathrm{CH}_{2}\right), 1.62\left(\mathrm{~m}, 2 \mathrm{H}, \mathrm{CH}_{2}\right), 0.90(\mathrm{t}, \mathrm{J}=7.3,3 \mathrm{H}$, $\mathrm{CH}_{3}$ ).

${ }^{13}$ C NMR (DMSO-d ) $\boldsymbol{\delta}: 156.38$ (C), 154.96 (C), 152.76 (CH-Ar), 150.38 (C), 149.92 (C), 141.21 (CH-Ar), 133.47 (C), 130.28 (2 x CH-Ar), 130.22 (2 x CH-Ar), 127.65 (CH-Ar), 119.22 (C), 42.62 $\left(\mathrm{CH}_{2}\right), 34.54\left(\mathrm{CH}_{2}\right), 26.85\left(\mathrm{CH}_{2}\right), 22.79\left(\mathrm{CH}_{2}\right)$, $22.36\left(\mathrm{CH}_{2}\right), 13.35\left(\mathrm{CH}_{3}\right)$.

Microanalysis: Theoretical: \%C: $57.85, \% \mathrm{H}$ : $5.62, \% \mathrm{~N}: 28.40$, Found: \%C: $57.46, \% \mathrm{H}: 5.58$, $\% \mathrm{~N}: 28.29$.

\section{Molecular modeling}

Docking studies were performed using MOE software [13] utilizing the homology model for $S$. aureus phenylalanine tRNA synthetase enzyme having phenylalanyl adenylate as a ligand [14]. Ligands were built using MOE and then the energy was minimized for each ligand, creating a ligand database. All minimizations were performed with MOE to a RMSD gradient of $0.01 \mathrm{Kcal} / \mathrm{mol} / \AA$ with MMFF94 forcefield, and partial charges were automatically calculated. The ligands were docked using the MOE default setting: Placement: Triangular Matcher, Rescoring 1: London $\Delta \mathrm{G}, 30$ poses were constructed for each compound and the best scoring model-ligand complexes were selected. The consequent ligand interactions within the constructed model were visualized using the MOE ligand interaction simulation. 


\section{Microbiological evaluation}

The synthesized compounds were evaluated for MIC against $S$. aureus SH1000 sensitive strain according to CSLI guidelines 2012 [15].

Moreover, compounds 11b, 16a, 17a and 17b were evaluated for their antimicrobial activity alongside comparator agent ampicillin (Amp) against a variety of clinically important pathogens. Isolates tested included clinical and NCTC/ATCC control organisms; $S$. aureus (including methicillin, tetracycline, erythromycin/clindamycin and vancomycin resistance), Klebsiella pneumoniae (including $3^{\text {rd }}$ generation cephalosporin and carbapenem resistance), Proteus mirabilis, Pseudomonas aeruginosa, Salmonella enteritidis, Acinetobacter baumannii, Enterococcus faecalis and faecium (including vancomycin resistance) and E. coli. Minimum Inhibitory Concentrations (MICs) were determined using microbroth dilution, the "gold standard", international standard ISO 10776$1[16]$.

P. aeruginosa PheRS and S. pneumonia PheRS enzymes assay

The inhibitory activity $\left(\mathrm{IC}_{50}\right)$ of the final compounds was determined using the tRNA aminoacylation assay adapted to a scintillation proximity assay (SPA) [17]. Test compounds were dissolved in $100 \%$ DMSO to a concentration of 3.3 $\mathrm{mM}$. To determine $\mathrm{IC}_{50}$ values the test compounds
$(2 \mu \mathrm{l})$ were serially diluted across 10 wells on the assay plates resulting in final assay concentrations ranging from $200 \mu \mathrm{M}$ to $0.4 \mu \mathrm{M}$. Briefly, the compounds were equilibrated by the addition of $33 \mu \mathrm{L}$ of the protein/substrate mix: $50 \mathrm{mM}$ Tris$\mathrm{HCl}(\mathrm{pH} 7.5), 8 \mathrm{mM} \mathrm{MgCl}, 1.25 \mathrm{mM}$ ATP, 1 $\mathrm{mM}$ spermine, $1 \mathrm{mM}$ DTT, $100 \mu \mathrm{M}\left[{ }^{3} \mathrm{H}\right] \mathrm{Phe}(75$ $\mathrm{cpm} / \mathrm{pmol}$ ), and $0.08 \mu M$ P. aeruginosa $\mathrm{PheRS}$ or $0.2 \mu M$ S. pneumonia PheRS. Control reactions contained only DMSO with no compound. This mixture was incubated at ambient temperature for $15 \mathrm{~min}$ and then reactions were started by the addition of $15 \mu \mathrm{l}$. coli tRNA $(80 \mu \mathrm{M}$ total tRNA or $2 \mu \mathrm{M}$ tRNA ${ }^{\text {Phe }}$ ), followed by incubation for $1 \mathrm{~h}$ at $37{ }^{\circ} \mathrm{C}$. Reactions were stopped by the addition of $5 \mu \mathrm{l}$ of $0.5 \mathrm{M}$ EDTA. $400 \mu \mathrm{g}$ of yttrium silicate (Ysi) poly-L-lysine coated SPA beads (Perkin-Elmer) in $150 \mu \mathrm{l}$ of $300 \mathrm{mM}$ citric acid were added and allowed to incubate at room temperature for $1 \mathrm{~h}$. The plates were analyzed using a 1450 Microbeta (Jet) liquid scintillation/ luminescent counter (Wallac). The curve fits and $\mathrm{IC}_{50}$ values were determined using the Sigmoidal Dose-Response Model in XLfit 5.3 (IDBS).

\section{Results and Discussion}

\section{Chemistry}

The sequence of the reactions followed in the preparation of the designed compounds is summarized in Schemes 1-3.

The synthesis of methyl esters (4a) and (4b)<smiles>c1cc2[nH]c3cc2c3c1</smiles>

$(1,2)$<smiles></smiles>

$(6 a, b)$<smiles>C=CC(=O)OC</smiles>

(3)

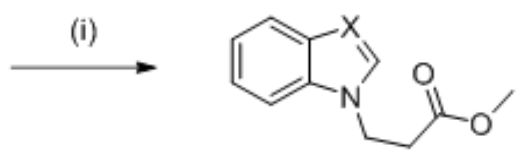

$(4 a, b)$<smiles>NNC(=O)CCn1c[X]c2ccccc21</smiles>

(5a, b)<smiles>[R]c1ccc(-c2csc(NNC(=O)CCn3c[X]c4ccccc43)n2)cc1</smiles>

Scheme 1. Reagents and conditions: (i) $\mathrm{DBU}, \mathrm{CH}_{3} \mathrm{CN}$, rt or $50^{\circ} \mathrm{C}, 6 \mathrm{~h}$ (ii) hydrazine monohydrate, $\mathrm{MeOH}, \mathrm{rt}, 3 \mathrm{~h}$ (iii) $\mathrm{KSCN}$, Conc. $\mathrm{HCl}$, MeOH, rt, overnight (iv) EtOH, reflux, overnight. 
were carried out via an aza-Michael addition reaction. Hydrazinolysis of the methyl ester compounds $\mathbf{( 4 a , b )}$ produced hydrazides [18] $\mathbf{( 5 a}, \mathbf{b})$ which were confirmed by the disappearance of the $\mathrm{CH}_{3}$ peak from both ${ }^{1} \mathrm{H}$ NMR and ${ }^{13} \mathrm{C}$ NMR spectra. ${ }^{1} \mathrm{H}$ NMR spectrum showed two singlet signals at $\sim 9.0 \mathrm{ppm}$ and $\sim 4.0 \mathrm{ppm}$ for the protons of $\mathrm{NH}$ and $\mathrm{NH}_{2}$ groups, respectively. Several attempts were investigated to convert (4a, b) directly to $(\mathbf{6 a}, \mathbf{b})$ without the hydrazinolysis step according to literature $[19,20]$. These trials involved refluxing the ester compounds $4 \mathbf{a}, \mathbf{b}$ with thiosemicarbazide in the presence of either acetone, EtOH with few drops of $\mathrm{AcOH}$ or $\mathrm{EtOH}$ with a few drops of dimethylsulfoxide (DMSO). However, all attempts were unsuccessful, based on ${ }^{1} \mathrm{H}$ NMR analysis. Therefore, an alternative pathway was taken, based on the reaction of the hydrazides $(\mathbf{5 a}, \mathbf{b})$ with $\mathrm{KSCN}$ through nucleophilic addition reaction [21]. Compounds 8a-c having 1,3-thiazole ring were achieved by the reaction of $6 \mathbf{a}$ or $6 \mathbf{b}$ and appropriate 2-bromo4'-substituted acetophenone (7a) or (7b) in EtOH under reflux overnight [22,23]. After column chromatography purification, it was found that ${ }^{1} \mathrm{H}$ NMR showed a few impurities. So, compound 8a was further purified by re-crystallization. However, the compound changed color during the heating of the re-crystallization process, and TLC showed several spots, indicating that the compound is heat sensitive. Preparative TLC was utilized for final purification of compound 8a. For compounds $\mathbf{8 b}$ and $\mathbf{8 c}$, fast column chromatography was done to avoid any decomposition in the desired compounds, then purified with preparative TLC. ${ }^{1} \mathrm{H}$ and ${ }^{13} \mathrm{C}$ NMR confirmed the structures with the singlet $\mathrm{CH}$ thiazole peak at approximately $7.5 \mathrm{ppm}$ in the ${ }^{1} \mathrm{H}$ NMR. The yields of this reaction were very low. Mass spectroscopy or microanalysis was not conducted for the structures of the prepared compounds owing to the instability (Scheme 1).

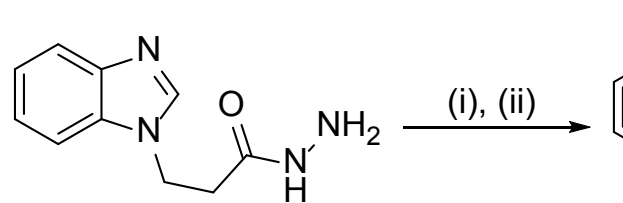

(5a)<smiles>CCn1cnc2ccccc21</smiles>

(9)<smiles>Cc1nnc(N)s1</smiles><smiles>[R]c1cc(C(=O)Cl)cc([R])c1[R]</smiles>

$(10 a, b)$

(iii)<smiles>[R]c1cc(C(=O)Nc2nnc(CCn3cnc4ccccc43)s2)cc([R3])c1[R2]</smiles>

$(11 a, b)$

Scheme 2. Reagents and conditions: (i) $\mathrm{KSCN}, \mathrm{HCl}, \mathrm{MeOH}$, rt, overnight (ii) Conc. $\mathrm{H}_{2} \mathrm{SO}_{4}$, $2 \mathrm{~h}$, aqueous $\mathrm{NH}_{3}$ (iii) $\mathrm{CH}_{2} \mathrm{C}_{12}, \mathrm{Et}_{3} \mathrm{~N}, \mathrm{O}^{\circ} \mathrm{C}-\mathrm{rt}$, overnight.

5-(2-(1H-benzimidazol-1-yl)ethyl)-1,3,4thiadiazol-2-amine (9) was achieved by the reaction of a methanolic solution of 3-(1H-benzimidazol1-yl)propane hydrazide (5a) with $\mathrm{KSCN}$ in acidic solution through a nucleophilic addition reaction [24,25]. The acidic condition afforded the cyclization of the 1,3,4-thiadiazole ring. ${ }^{1} \mathrm{H}$ NMR spectrum showed downfield singlet signal at $7.03 \mathrm{ppm}$ corresponding to the two protons of the primary amino group and more carbon atom at $154.69 \mathrm{ppm}$ appeared in ${ }^{13} \mathrm{C}$ NMR spectrum. The synthesis of 11a, b was achieved through the nucleophilic substitution reaction of 9 with benzoyl chloride derivatives (10a) or (10b) in $\mathrm{CH}_{2} \mathrm{Cl}_{2} / \mathrm{Et}_{3} \mathrm{~N}$. The low product yield was presumably owing to the reduced nucleophilicity of the primary amino group (Scheme 2).

Thiosemicarbazide $(\mathbf{1 2}, \mathbf{1 3})$ cyclization in alkaline medium resulted in the formation of 1,2,4-triazoles $(\mathbf{1 4}, \mathbf{1 5})$. This is because $\mathrm{N}-4$, in alkaline medium, is more nucleophilic than the sulfur of the thiocarbonyl group and oxygen of carbonyl group producing 1,2,4-trizoles [26-28]. Treatment of 14 or 15 with potassium carbonate as a base and an appropriate alkyl halide gave the desired thioethers (16 and 17) in very good yields (Scheme 3). 


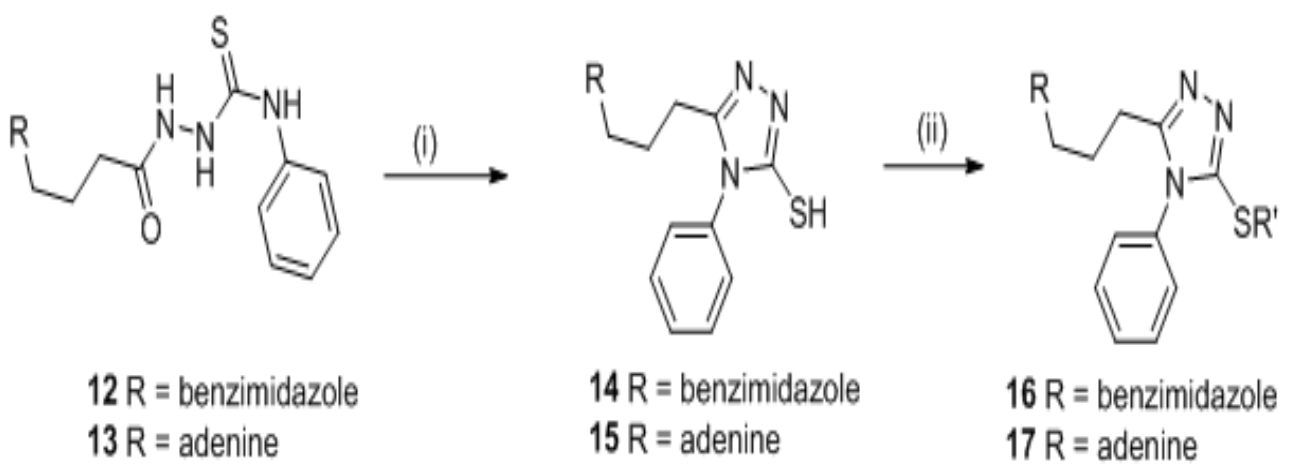

Scheme 3. Reagents and conditions: (i) (a) $2 \mathrm{~N} \mathrm{NaOH}, 5$ h, rt, (b) Conc. HCl (ii) Alkyl halide, anhyd. K2CO3, DMF, rt, overnight.

\section{Molecular modeling evaluation}

These series were developed to include either adenine or a biarylmimic (benzimidazole or indole) to represent the adenyl moiety of phenylalanyl adenylate (Table 1). The 'adenyl' portion was linked, through a 3-5 atom linker that spans the hydrophobic channel, to a heterocyclic 5-membered ring having either thiol or nitrogen or both to make H-bonds with the key binding amino acid residues (His172, Ser174, Gln214 and/or Glu216), and finally the remainder of the compound, which may be aliphatic or aromatic, to fill the large hydrophobic pocket and may contributed with $\mathrm{H}$-bond interactions.
The compounds were investigated through docking studies. The thiol group, amino group and/ or carbonyl group for compounds 8a - c showed interactions with the following binding residues in the active site: Ser174, Gln214 and Gly288. The amide group in compounds 11a and 11b interacted with Ser174 and Ala311 and the thiol group of 1,3,4-thiadiazolyl moiety interacted with His 172. As observed for compounds 16a, 17a and $\mathbf{1 7} \mathbf{b}$, the thiol group formed a $\mathrm{H}$-bond with acidic Glu216 and for compound 16b, Gln214 formed a $\mathrm{H}$-bond with the 1,2,4-triazole moiety (Table 2).

TABLE 1. 3D and 2D models of binding interactions of phenylalanyl-adenylate in S. aureus PheRS activE SITE ${ }^{[14]}$
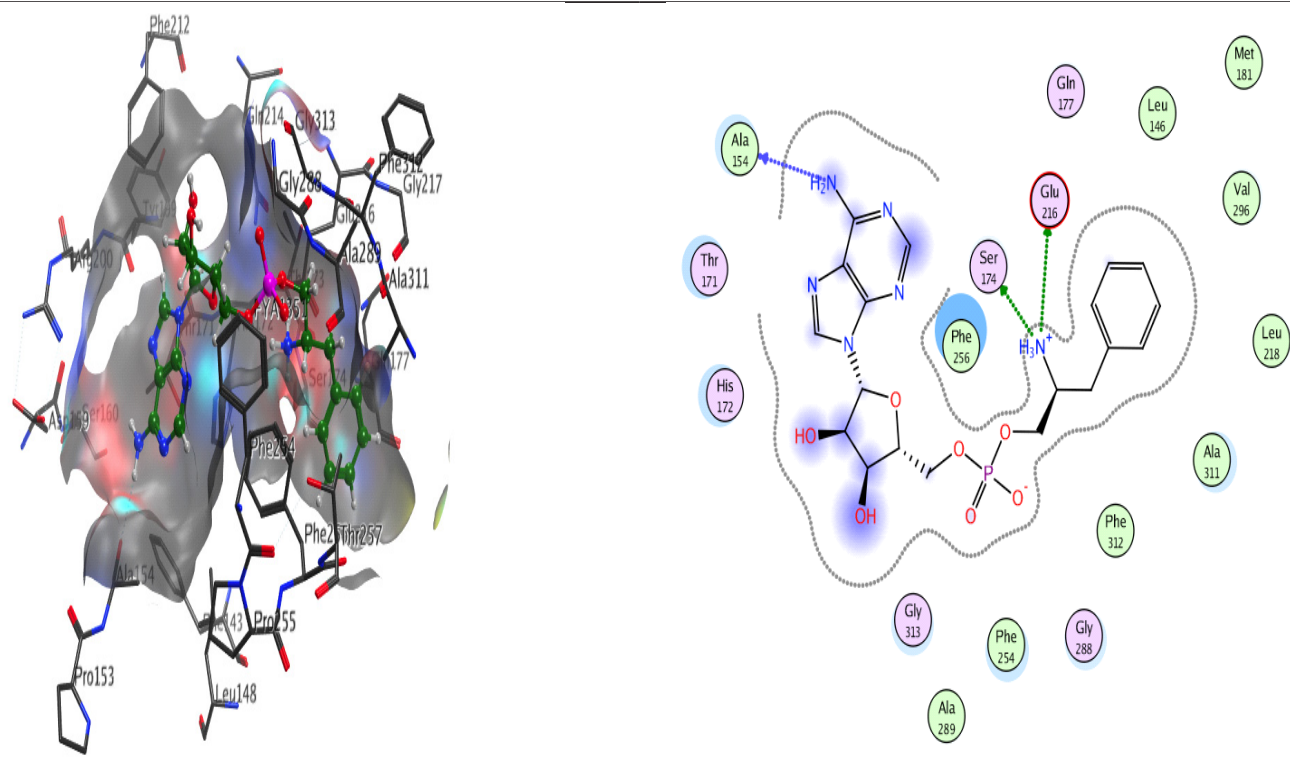
TABLE 2. 2D models of binding interactions of compounds in $S$. aureus PheRS active site using MOE

(Pn:

(영 (연

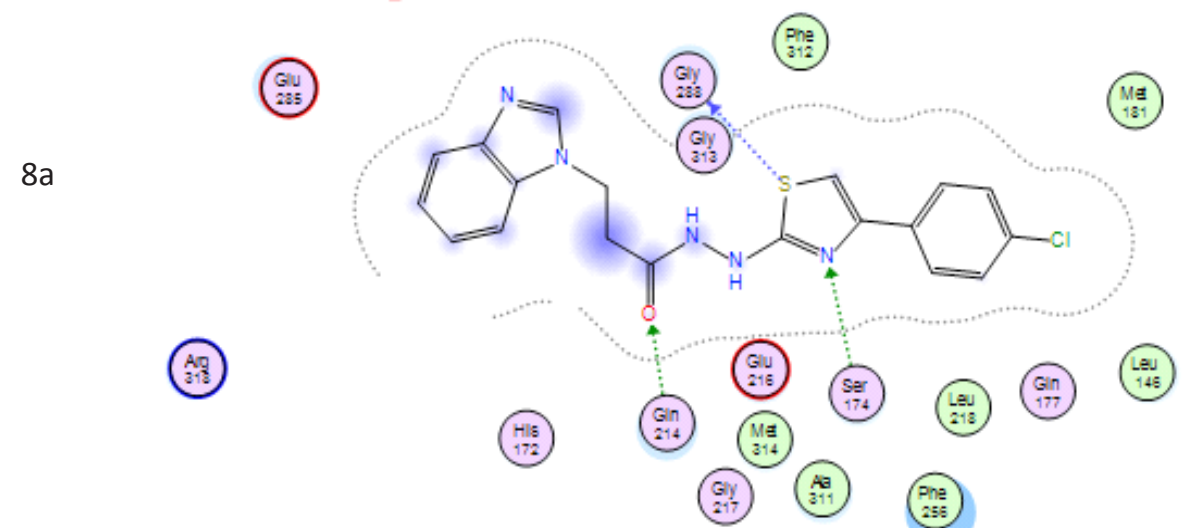

$\left(\begin{array}{ccc}2 \mathrm{n} 2 \\ 254\end{array}\right)$

(영 (अ)

윰

$8 b$

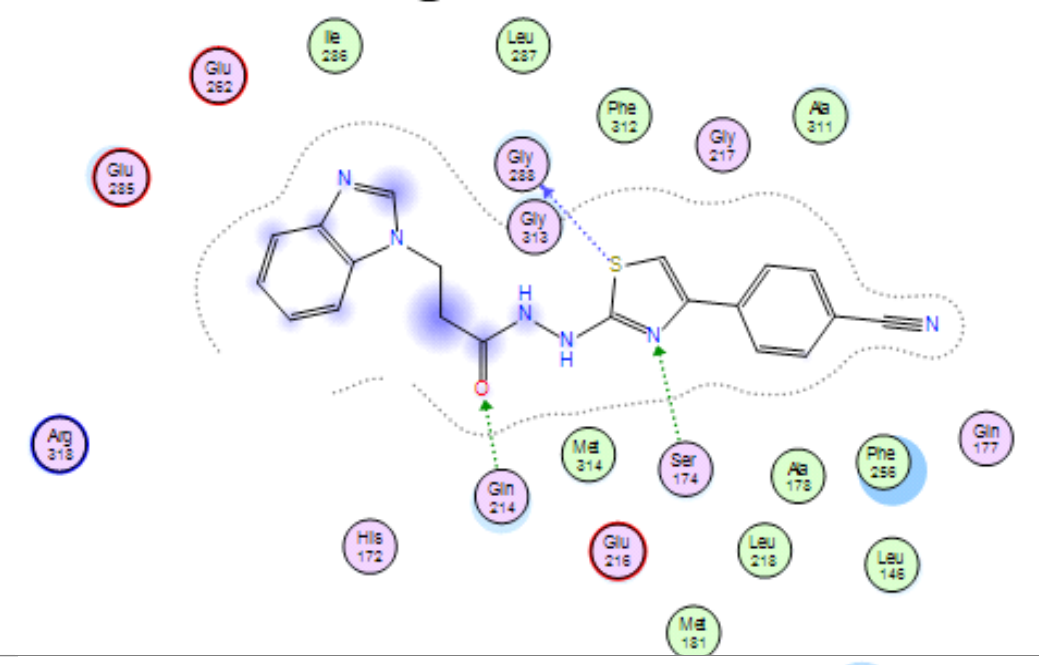

(va)
(5尺)
(Gi1) (iㅏ)
(이감
Phe

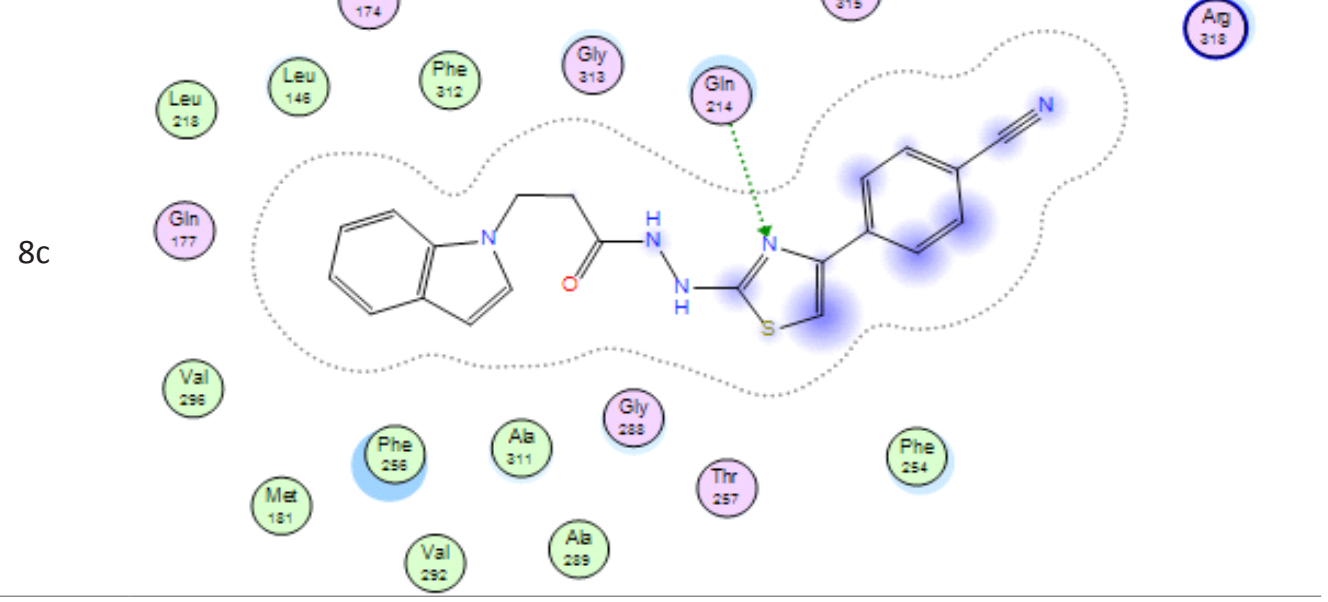

Egypt.J.Chem. Special Issue (2018) 


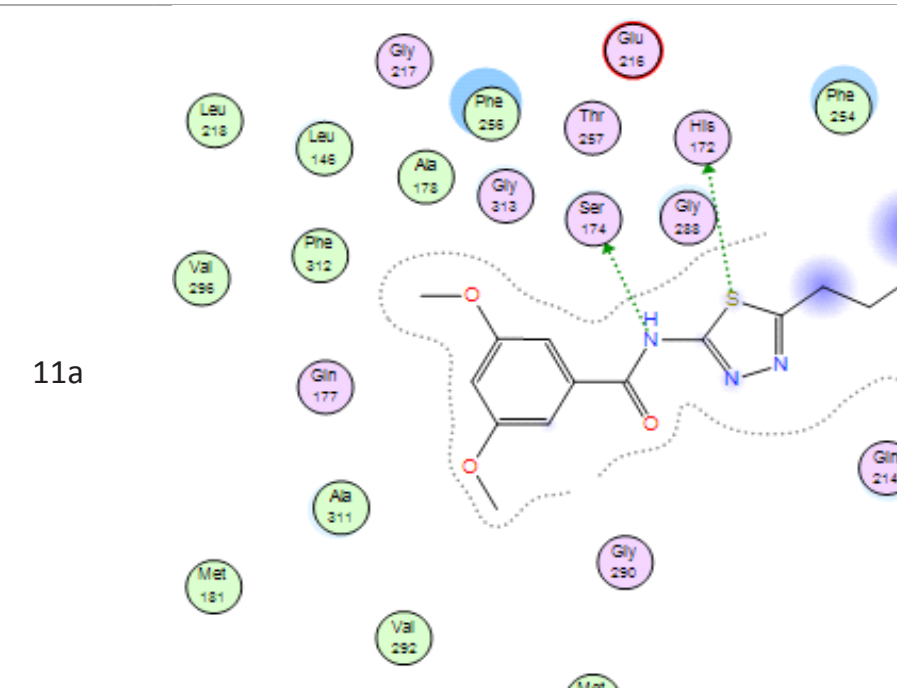

คำ

(Mat)

(Gin

$11 b$

A 190

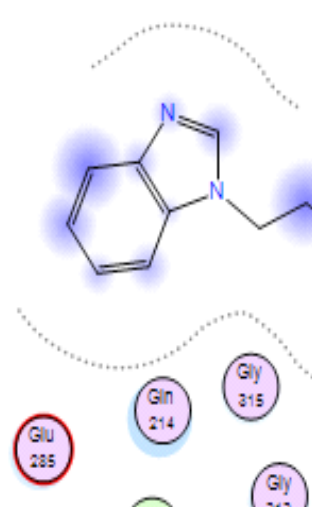

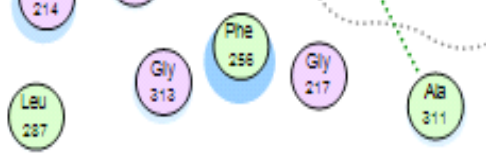

(M: (14)

$\left(\begin{array}{c}716 \\ 172\end{array}\right)$

Gi1

(aㅕ)

(aㅗ)

(6119) (197) (1919)

(18)

(G19)

(iㅣ)

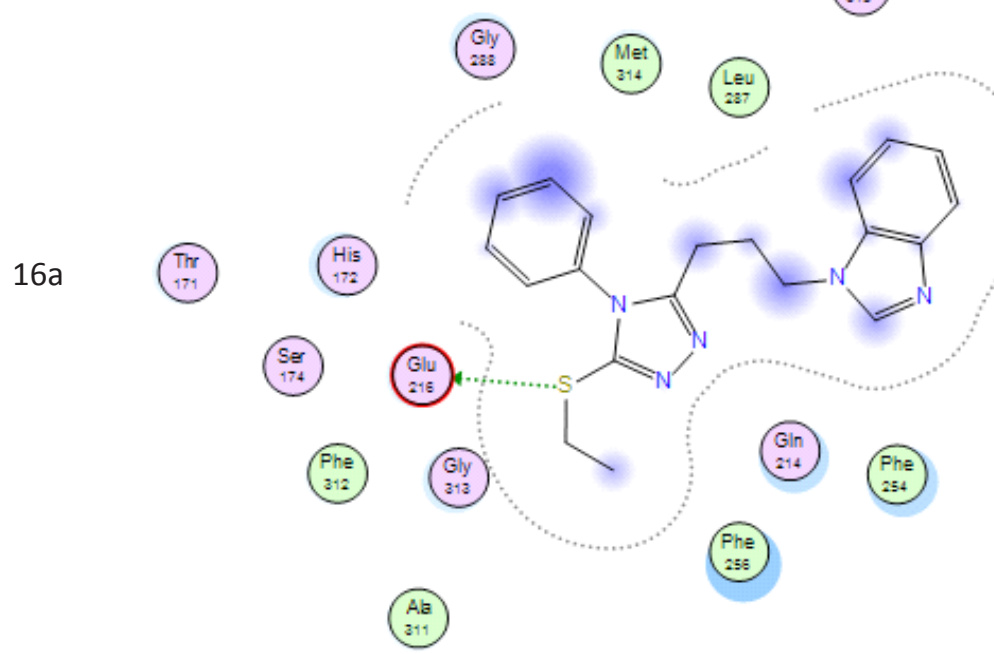

(49) 


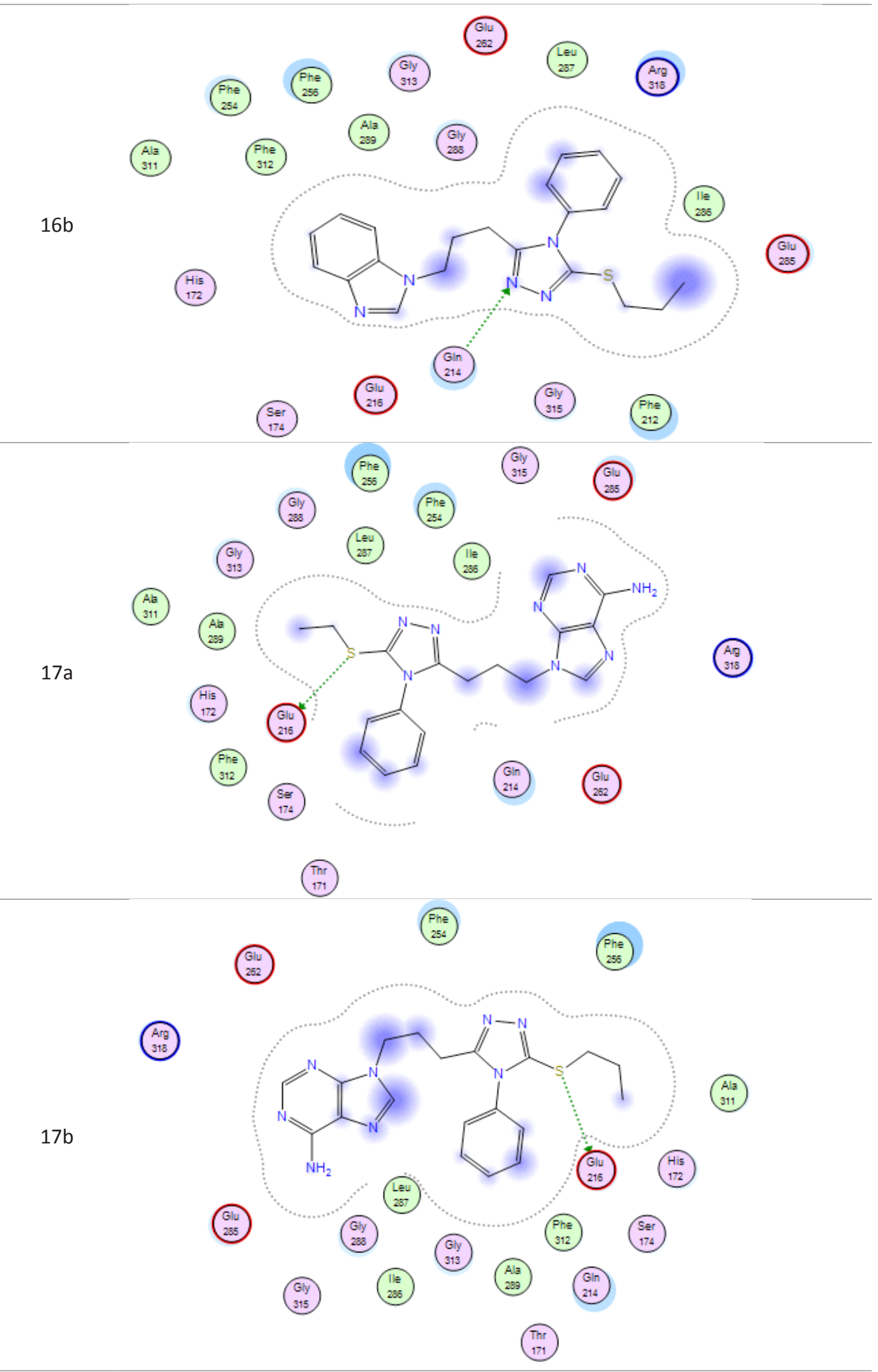

Egypt.J.Chem. Special Issue (2018) 
Microbiological evaluation

Compounds 8a-c were not subjected to microbiological evaluation because of their instability. Nevertheless, none of the tested compounds showed inhibitory activity (MIC $\geq$ $128 \mu \mathrm{g} / \mathrm{mL}$ ) against $S$. aureus $\mathrm{SH} 1000$ sensitive strains. The synthesized compounds were microbiologically tested for $P$. aeruginosa PheRS and S. pneumonia PheRS inhibition through aminoacylation assay due to the availability of these enzymes and their high degree of similarity with $S$. aureus PheRS. Analysis of the synthesized

TABLE 3. MIC $(\mu \mathrm{g} / \mathrm{mL})$ values against $S$. aureus sensitive $\mathrm{SH} 1000$ strain and $\mathrm{IC}_{50}$ data against $S$. pneumonia PheRS and $P$. aeruginosa PheRS ( $\mu \mathrm{M})$. series resulted in only compound 34a $\left(\mathrm{IC}_{50} 199\right.$ $\mu \mathrm{M})$ exhibiting moderate inhibitory activity (Table 3).

Moreover, compounds (11b, 16a, 17a and 17b) showed no inhibitory activity (MIC $\geq 128$ $\mu \mathrm{g} / \mathrm{mL}$ ) against the tested bacteria. However, moderate inhibitory activity $(32-64 \mu \mathrm{g} / \mathrm{mL})$ was observed with 11b and 17a against E. faecalis sensitive and vanA and vanB resistant strains (Table 4).

\begin{tabular}{|c|c|c|c|}
\hline & $\begin{array}{c}\text { MIC }(\mu \mathrm{g} / \mathrm{mL}) \\
\text { S. aureus Sensitive } \\
\text { SH1000 }\end{array}$ & $\begin{array}{l}\text { S. pneumonia } \\
\text { PheRS IC }_{50}(\mu \mathrm{M})\end{array}$ & $\begin{array}{l}\text { P. aeruginosa } \\
\text { PheRS IC }{ }_{50}(\mu \mathrm{M})\end{array}$ \\
\hline $11 a$ & $>128$ & 345 & 742 \\
\hline $11 b$ & $>128$ & 685 & 907 \\
\hline $16 a$ & $>128$ & 561 & 343 \\
\hline $16 \mathrm{~b}$ & $>128$ & 390 & 375 \\
\hline $17 a$ & $>128$ & 619 & 199 \\
\hline $17 b$ & $>128$ & 686 & 294 \\
\hline
\end{tabular}

TABLE 4. Broad microbiological evaluation of 11b, 16a, 17a and 17b (MIC ( $\mu \mathrm{g} / \mathrm{mL})$ determination).

\begin{tabular}{|c|c|c|c|c|c|}
\hline Organism & Amp & $11 b$ & $16 a$ & $17 a$ & $17 b$ \\
\hline S. aureus ATCC 29213 sensitive & 4 & $>128$ & $>128$ & $>128$ & $>128$ \\
\hline $\begin{array}{l}\text { S. aureus NCTC } 12493 \text { mecA } \\
\text { resistant }\end{array}$ & $>128$ & 128 & $>128$ & 128 & $>128$ \\
\hline K. pneumoniae 21856 sensitive & $>128$ & $>128$ & $>128$ & $>128$ & $>128$ \\
\hline $\begin{array}{l}P . \quad \text { mirabilis NCTC } 10975 \\
\text { sensitive }\end{array}$ & $>128$ & $>128$ & $>128$ & $>128$ & $>128$ \\
\hline $\begin{array}{l}P . \text { aeruginosa ATCC } 27853 \\
\text { sensitive }\end{array}$ & $>128$ & $>128$ & $>128$ & $>128$ & $>128$ \\
\hline S.enteritidis 8204 sensitive & 8 & $>128$ & $>128$ & $>128$ & $>128$ \\
\hline A.baumannii572 sensitive & $>128$ & $>128$ & $>128$ & $>128$ & $>128$ \\
\hline $\begin{array}{l}\begin{array}{l}\text { B.cepacia } \\
\text { sensitive }\end{array} \\
\text { NCTC }\end{array}$ & $>128$ & $>128$ & $>128$ & $>128$ & $>128$ \\
\hline $\begin{array}{l}\text { E. faecalisATCC } 29212 \\
\text { sensitive }\end{array}$ & 2 & 64 & $>128$ & $>128$ & $>128$ \\
\hline $\begin{array}{l}\text { E. faecalis NCTC } 12201 \text { vanA } \\
\text { resistant }\end{array}$ & 16 & 32 & $>128$ & 64 & $>128$ \\
\hline $\begin{array}{l}\text { E. faecalis ATCC } 51299 \text { vanB } \\
\text { resistant }\end{array}$ & 8 & 64 & $>128$ & 64 & $>128$ \\
\hline E. faecium 16568 sensitive & 4 & - & $>128$ & - & $>128$ \\
\hline E. coli ATCC 25922 sensitive & 8 & $>128$ & $>128$ & $>128$ & $>128$ \\
\hline
\end{tabular}




\section{Conclusion}

In summary, three novel series were designed and synthesized depending on the natural substrate, phenylalanyl-adenylate. All designed compounds make H-bonds with the key amino acid residues allowing the orientation of the compounds in the adenylate and amino acid binding sites. As the compounds in thiazole series are unstable, the future work should be optimized for the structure for further investigation of compound stability and yield. Analysis of the thiadiazole and triazole series resulted in only compound 17a $\left(\mathrm{IC}_{50} 199\right.$ $\mu \mathrm{M})$ exhibiting moderate inhibitory activity against $P$. aeruginosa PheRS. Compounds 11b and 17 a showed moderate inhibitory activity (32$64 \mu \mathrm{g} / \mathrm{mL}$ ) against $E$. faecalis sensitive and vanA and vanB resistant strains.

\section{Conflict of Interest}

The authors report that they have no conflict of interest to declare.

\section{Acknowledgments}

We thank the Egyptian Government for a Channel research scholarship to SSE and the EPSRC Mass Spectrometry Centre, Swansea, U.K. for mass spectroscopy data. The authors are grateful for the financial support to JMB provided by the National Institutes of Health (grant number: 1SC3GM098173-01A1).

\section{References}

1. Tong, S. Y., Davis, J. S., Eichenberger, E., Holland, T. L., Fowler, V. G., Staphylococcus aureus infections: Epidemiology, pathophysiology, clinical manifestations, and management. Clinical Microbiology Reviews, 28, 603-661 (2015).

2. Durhan, E., Korcan, S. E., Altindis, M., Konuk, M., Fitness and competitive growth comparison of methicillin resistant and methicillin susceptible Staphylococcus aureus colonies. Microbial Pathogenesis, 106, 69-75 (2017).

3. Lee, J. H., Methicillin (oxacillin)-resistant Staphylococcus aureus strains isolated from major food animals and their potential transmission to humans. Applied and Environmental Microbiology, 69, 6489-6494 (2003).

4. Ibba, M., Söll, D., Aminoacyl-tRNA synthesis. Annual Review of Biochemistry, 69, 617-650 (2000).

5. Carter Jr, Cognition C. W., Mechanism, and evolutionary relationships in aminoacyltRNA synthetases. Annual Review of Biochemistry, 62, 715-748 (1993).

6. Hurdle, J. G., O’Neill, A. J., Chopra, I., Prospects for aminoacyl-tRNA synthetase inhibitors as new antimicrobial agents. Antimicrobial Agents and Chemotherapy, 49, 4821-4833 (2005).

7. Keller, B., Kast, P., Hennecke, H., Cloning and sequence analysis of the phenylalanyltRNA synthetase genes (pheST) from Thermus thermophilus. FEBS Letters, 301, 83-88 (1992).

8. Kreutzer, R., Kruft, V., Bobkova, E. V., Lavrik, O. I., Sprinzl, M., Structure of the phenylalnyl-tRNA synthetase genes from Thermus thermophilus HB8 and their expression in Escherichia coli. Nucleic acids Research, 20, 4173-4178 (1992).

9. Yeom, C.-E., Kim, M. J., Kim, B. M. 1., 8-Diazabicyclo [5.4. 0] undec-7-ene (DBU)promoted efficient and versatile aza-Michael addition. Tetrahedron, 63, 904-909 (2007).

10. Demirayak, Ş., Benkli, K., Güven, K., Synthesis and antimicrobial activities of some 3-arylamino-5-[2-(substituted 1-imidazolyl) ethyl]-1, 2, 4-triazole derivatives. European Journal of Medicinal Chemistry, 35, 10371040 (2000).

11. Zhou, M., Eun, Y.-J., Guzei, I. A., Weibel, D. B., Structure-activity studies of divin: An inhibitor of bacterial cell division. ACS Medicinal Chemistry Letters, 4, 880-885 (2013).

12. Li, J. H., Snyder, J. K., Pyrrole as a dienophile in intramolecular inverse electron-demand Diels-Alder reactions with 1, 2, 4-triazines. The Journal of Organic Chemistry, 58, 516519 (1993).

13. Molecular Operating Environment (MOE 2014.0901) Chemical Computing Group Inc, Montreal Quebec Canada http://www. chemcomp.com. 2014.0901.

14. Elbaramawi, S. S., Ibrahim, S. M., Lashine, E.-S. M., El-Sadek, M. E., Mantzourani, E.,

Egypt.J.Chem. Special Issue (2018) 
Simons, C., Exploring the binding sites of Staphylococcus aureus phenylalanine tRNA synthetase: A homology model approach. Journal of Molecular Graphics and Modelling, 73, 36-47 (2017).

15. CSLI. Methods for Dilution Antimicrobial Susceptibility Tests for Bacteria That Grow Aerobically, Approved Standard - Ninth Edition. CSLI document M07-A9. Wayne. PA: Clinical and Laboratory Standards Institute (2012).

16. ISO/FDIS. 20776-1. International Organization for Standardization, Clinical laboratory testing and in vitro diagnostic test systems -- Susceptibility testing of infectious agents and evaluation of performance of antimicrobial susceptibility test devicesPart 1: Reference method for testing the in vitro activity of antimicrobial agents against rapidly growing aerobic bacteria involved in infectious diseases (2006).

17. Macarrón, R., Mensah, L., Cid, C., Carranza, C., Benson, N., Pope, A. J., Díez, E., A homogeneous method to measure aminoacyl-tRNA synthetase aminoacylation activity using scintillation proximity assay technology. Analytical Biochemistry, 284, 183-190 (2000).

18. El-Saied, F., Al-Hakimi, A., Wahba, M., Shakdofa, M., Preparation, Characterization and Antimicrobial Activities of N'-((3(hydroxyimino) butan-2-ylidene)-2 (phenylamino) acetohydrazide and Its Metal Complexes. Egyptian Journal of Chemistry, 60, 1-24 (2017).

19. Kumawat, M., Talesara, G. L., Synthesis of ethoxyphthalimido derivatized thiadizole assembled imidazolidinone and chloroazetidinone systems from common intermediate Schiff's bases and evaluation of their antibacterial activity. Journal of Applicable Chemistry, 2, 754-764 (2013).

20. Idhayadhulla, A., Kumar, R. S., Nasser, A. J. A., Kavimani, S., Indhumathy, S., Anti-Inflammatory Activity of New Series of 1, 4-Dihydropyridine Derivatives. Pharmaceutical Chemistry Journal, 49, 463466 (2015).

21. Murty, M., Ram, K. R., Rao, B. R., Rao, R. V., Katiki, M. R., Rao, J. V., Pamanji, R., Egypt.J.Chem. Special Issue (2018)
Velatooru, L., Synthesis, characterization, and anticancer studies of $\mathrm{S}$ and $\mathrm{N}$ alkyl piperazine-substituted positional isomers of 1, 2, 4-triazole derivatives. Medicinal Chemistry Research, 23, 1661-1671 (2014).

22. Samshuddin, S., Narayana, B., Sarojini, B. K., Shetty, D. N., Suchetha Kumari, N., Synthesis, characterization, and biological evaluation of some new functionalized terphenyl derivatives. International Journal of Medicinal Chemistry, 2012, 1-13 (2012).

23. Badr, S., Synthesis and antiinflammatory activity of novel 2, 5-disubstituted thiophene derivatives. Turkish Journal of Chemistry, 35, 131-143 (2011).

24. Manjunatha, K., Poojary, B., Kumar, V., Lobo, P. L., Fernandes, J., Chandrashekhar, C., Synthesis, characterization and antimicrobial activities of imidazo-[2, 1, b] [1, 3, 4]-thiadiazoles. Der Pharma Chemica, 7, 207-215 (2015)

25. Hafez, H. N., Microwave-Assisted Synthesis and Cytotoxicity Evaluation of Some Novel Pyrazole Containing Imidiazoles, Pyrazoles, Oxazoles, Thiadiazoles and Benzochromene Derivatives. Egyptian Journal of Chemistry, 60, 1015-1028 (2017).

26. Coruh, I., Rollas, S., Turan, S. O., Akbuga, J., Synthesis and evaluation of cytotoxic activities of some 1, 4-disubstituted thiosemicarbazides, 2, 5-disubstituted-1, 3, 4-thiadiazoles and 1, 2, 4-triazole-5thiones derived from benzilic acid hydrazide. Marmara Pharmaceutical Journal, 16, 56-63 (2012).

27. Abdel-Fattah, H. A., El-Etrawy, A. S., Gabr, N. R. M., Synthesis and biological evaluation of some new 1, 3, 4-oxa, thiadiazole and 1, 2, 4-triazole derivatives attached to benzimidazole. International Journal of Pharmaceutical Chemistry, 4, 112-118 (2014).

28. Khidre, R. E., Radini, I. A. M., AbdelWahab, B. F., Synthesis of New Heterocycles Incorporating 3-(N-phthalimidomethyl)-1, 2, 4-triazole as Antimicrobial Agents. Egyptian Journal of Chemistry, 59, 731-744 (2016).

(Received 12/6/2018; accepted 10/7/2018) 


\section{تصميم و تثييا وتقييم مركبات جديدة تعمل كمثبطات لمخلق فينيل الانين الحمض الريبى

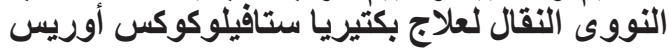

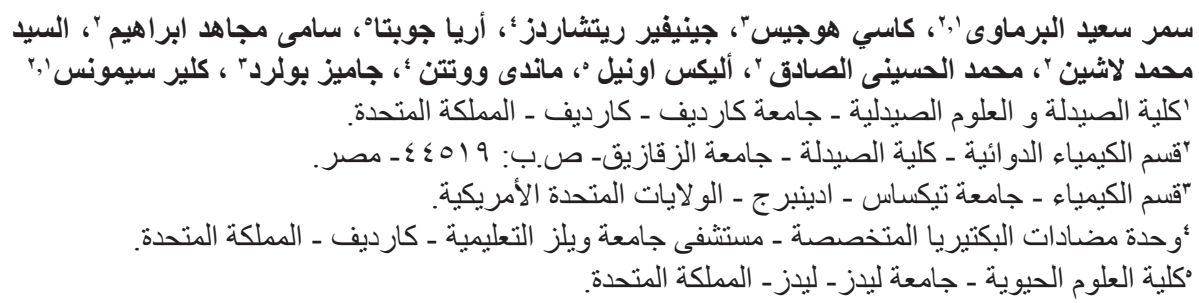

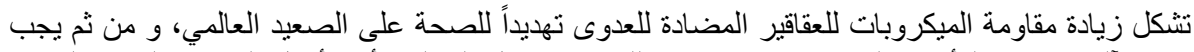

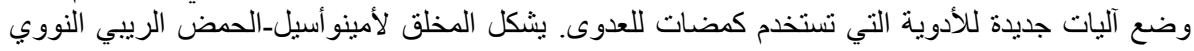

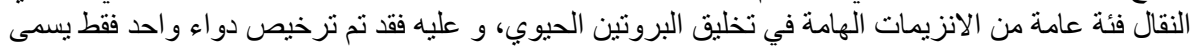

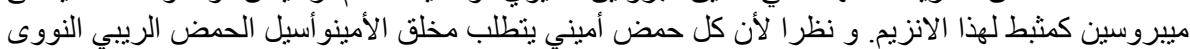

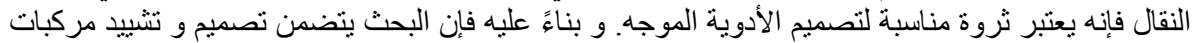

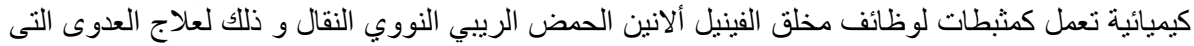

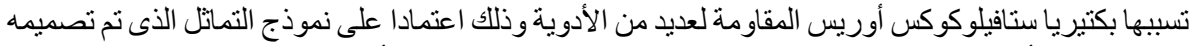

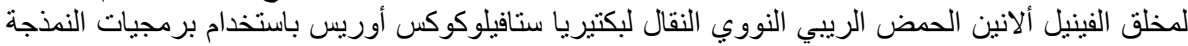

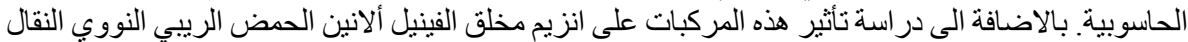

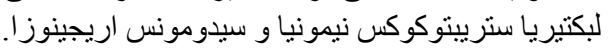

